\title{
Des méthodes d'intégration par arcs de sections coniques aux échelles de modules. Legendre lecteur de Landen*
}

\author{
Ivahn Smadja \\ Université Paris Diderot - Paris 7 - UMR 7219
}

\begin{abstract}
Résumé
Dans cet article, nous analysons en détail les deux mémoires de Legendre, Sur les intégrations par arcs d'ellipse (1786), en comparant les deux démonstrations qu'il donne de la rectification de l'hyperbole au moyen de deux arcs d'ellipse. Entre le premier et le second mémoire, Legendre prend connaissance d'un théorème analogue de Landen, qu'il cherche à incorporer à son projet de constitution d'un calcul des arcs d'ellipse et dont il propose une interprétation originale en le déduisant de ses propres principes. En retraçant la préhistoire de la transformation de Landen, des premières méthodes d'intégration par arcs de sections coniques chez Maclaurin, d'Alembert et Landen jusqu'à l'élaboration par Legendre des échelles de modules, nous cherchons à mettre en lumière les conditions de son identification. Nous montrons comment Legendre fut amené à discerner dans le théorème de Landen ce qui entre ses mains allait devenir l'instrument privilégié de la mise en ordre systématique des transcendantes elliptiques, grâce à une lecture orientée par les exigences spécifiques liées à une méthode d'approximation fondée sur la variation continue des paramètres et à la constitution de tables numériques d'arcs d'ellipse.
\end{abstract}

\section{Introduction}

Le Traité des fonctions elliptiques (1825) s'ouvre sur une définition précise du projet qui conduisit Legendre à la classification des transcendantes elliptiques sous la forme définitive à laquelle il parvint plusieurs décennies après ses premiers travaux sur les méthodes d'intégration par arcs d'ellipse. La théorie des intégrales elliptiques y est présentée comme un palier intermédiaire accessible dans la poursuite d'un programme plus vaste visant à étendre le calcul intégral à toutes les transcendantes susceptibles de se présenter dans l'analyse ${ }^{1}$. L'organisation d'ensemble de la théorie de la maturité atteste le plein succès de cette entreprise par l'arrangement en damier des

\footnotetext{
${ }^{*}$ Classification mathématique par sujets (2000) : 01A50, 33E05

${ }^{1}$ Cf. [50, Introduction, p. 1] : "Si [écrit Legendre] on pouvait ranger dans un ordre méthodique les diverses transcendantes qui n'ont été connues et employées jusqu'ici que sous le nom de quadratures; si en étudiant leurs propriétés on trouvait les moyens de les réduire aux expressions les plus simples dont elles sont susceptibles dans l'état de généralité, et d'en calculer avec facilité les valeurs approchées lorsqu'elles deviennent entièrement déterminées; alors les transcendantes dont il s'agit, désignées chacune par un caractère particulier et soumises à un algorithme convenable, pourraient être employées dans l'analyse à peu près comme le sont les arcs de cercle et les logarithmes; les applications du calcul intégral ne seraient plus arrêtées, comme elles l'ont été jusqu'ici, par cette espèce de barrière qu'on ne tente plus de franchir, lorsque le problème est ramené aux quadratures, et les solutions, à peine commencées par cette réduction, recevraient tous les développements que comporte la nature de la question. Ce qu'il serait comme impossible d'exécuter dans un plan aussi vaste que celui qui vient d'être tracé, on peut au moins le réaliser à l'égard des transcendantes qui se rapprochent le plus des fonctions circulaires et logarithmiques, telles les arcs d'ellipse et d'hyperbole, et en général les transcendantes auxquelles nous avons donné le nom de fonctions elliptiques."
} 
transcendantes elliptiques, ordonnées conjointement selon deux échelles de modules distinctes, de manière à ménager la libre circulation d'une expression à l'autre en fonction des besoins du calcul. L'assignation de ce but ultime conférait toutefois à la démarche son unité et sa cohérence, dès la première ébauche d'un calcul des arcs d'ellipse. Si, comme Legendre le souligne lui-même à de multiples reprises, les arcs de cercle et les logarithmes suffisent au calcul d'un grand nombre d'intégrales, pour s'acquitter de certaines autres en revanche, il est nécessaire d'avoir recours à de nouvelles transcendantes, parmi lesquelles les arcs d'ellipse semblent de prime abord s'imposer à l'attention du mathématicien comme les plus simples. Dans les deux mémoires Sur les intégrations par arcs d'ellipse (1786), Legendre cherche donc à constituer les arcs d'ellipse en un "nouvel instrument de calcul" ${ }^{2}$ dont la portée est d'autant plus grande qu'il montre que les arcs d'hyperbole peuvent s'y réduire. Cependant certains problèmes pour lesquels les arcs d'ellipse sont à leur tour insuffisants, tels la surface du cône oblique ou le mouvement de rotation d'un corps qui n'est sollicité par aucune force accélératrice, requièrent encore une nouvelle extension, pour autant qu'ils dépendent en général d'intégrales de la forme $\int \frac{P d x}{R}$ dans laquelle $P$ est une fonction rationnelle de $x$, et $R$ la racine carrée d'un polynôme du quatrième degré. Le Mémoire sur les transcendantes elliptiques (1793) est alors consacré à l'étude systématique de ces intégrales dont les propriétés justifient que Legendre les réunisse en un seul ordre, quoiqu'il y distingue trois espèces de transcendantes, ordonnées de la plus simple à la plus composée. Des considérations de simplicité analytique conduisent ainsi à reconnaître que, contrairement aux apparences, les arcs d'ellipse ne sont pas les transcendantes les plus simples après les arcs de cercle et les logarithmes et qu'une autre transcendante, la fonction elliptique de première espèce, aurait de fait plus de titres à faire valoir à cet égard. Enfin, dans les Exercices de calcul intégral (1811), puis dans le Traité des fonctions elliptiques (1825), qui reprend et prolonge le traité précédent, Legendre étend le calcul à la classe entière des transcendantes elliptiques en utilisant pleinement les possibilités qu'offrent certaines transformations remarquables, au premier rang desquelles la transformation dont il attribue la découverte à Landen, quoiqu'il revendique pour lui-même le mérite d'avoir montré l'usage qui pouvait en être fait pour atteindre les fins qu'il se proposait.

Le projet de Legendre induisait toutefois une lecture orientée des recherches antérieures, et il est à cet égard significatif que le mathématicien français ait pris soin, en brossant lui-même une histoire succincte de son sujet ${ }^{3}$, de signaler parmi les travaux de ses prédécesseurs les résultats qu'il jugeait rétrospectivement pertinents pour la perspective qu'il adoptait. Par les choix qu'elle opère, les filiations qu'elle dessine et la structuration du champ ${ }^{4}$ qu'elle propose, la présentation historique de Legendre a très largement contribué à façonner l'historiographie ultérieure, bien qu'elle soit loin d'être neutre. Parmi les traits les plus saillants qui la caractérisent en effet, notons que Legendre marque tout d'abord nettement une opposition entre une préhistoire qui se caractériserait par une accumulation de résultats disparates "ne pouv[ant] former aucune théorie" (Maclaurin, d'Alembert) et un avènement auquel le résultat de Landen sur la rectification de

\footnotetext{
${ }^{2}[48$, p. 4$]$.

${ }^{3}$ Nous reproduisons en annexe dans son intégralité le texte des présentations historiques que Legendre donne dans les Exercices et dans le Traité, en indiquant les différences entre la première et la seconde version.

${ }^{4}$ Sur la notion de champ et son utilisation en histoire des mathématiques, cf. [27, p. 53]. Catherine Goldstein et Norbert Schappacher empruntent ce concept à la sociologie de Pierre Bourdieu en mettant en avant le fait que, dans le cas de l'histoire des mathématiques, les mathématiciens se font eux-mêmes historiens de leur propre discipline et contribuent à la constitution d'un champ en ménageant notamment dans leurs œuvres tout un jeu complexe de références aux œuvres antérieures. Les auteurs s'appuient à cet égard sur la citation suivante tirée du texte d'une conférence de 1976 : "Un des indices les plus sûrs de la constitution d'un champ est, avec la présence dans l'œuvre de traces de relations objectives ...aux autres œuvres, passées ou contemporaines [du champ], l'apparition d'un corps de conservateurs de vies...ou des ouvres. ....Et un autre indice du fonctionnement en tant que champ est la trace de l'histoire du champ dans l'œeuvre" [3, p. 116-117]. Les présentations historiques qui ouvrent les traités de Legendre semblent réunir les principales de ces caractéristiques.
} 
l'hyperbole préparait, quoique ce dernier n'ait pas su voir tout le parti qu'il aurait été fondé à en tirer. Legendre recourt en effet à des tournures conditionnelles pour évoquer Landen, en soulignant les possibilités que celui-ci aurait ouvertes sans toutefois les reconnaître comme telles ${ }^{5}$. Christian Gilain ([25, p. XXIX-XXXI]) a montré de manière détaillée en quoi cette présentation pouvait conduire à d'importantes distorsions non seulement en omettant de distinguer les apports respectifs de Maclaurin et de d'Alembert, mais aussi en minorant systématiquement la contribution de ce dernier faute d'en saisir la cohérence propre. Outre cette première césure constitutive du champ, Legendre en marque une seconde en soulignant l'importance des mémoires de Fagnano et d'Euler relatifs aux théorèmes d'addition des intégrales elliptiques. Bien qu'il se réfère ainsi à ce qu'il présente comme deux courants de recherche relativement indépendants, il concède néanmoins, fût-ce de manière implicite, que ces courants s'inscrivent dans une même trame historique. Fagnano par exemple est salué à la fois pour la duplication de l'arc de lemniscate et pour son théorème sur les arcs d'ellipse ou d'hyperbole dont la différence est exprimable comme une quantité algébrique ${ }^{6}$, quoique rien ne soit dit sur le point de savoir comment ces premiers travaux de Fagnano pourraient se rapporter aux seconds. Euler ensuite aurait eu, toujours selon Legendre, le mérite de dégager la forme générale des théorèmes d'addition en comparant les arcs d'une même ellipse, d'une même hyperbole ou d'une même lemniscate, et plus généralement toutes les transcendantes qui rentrent dans la formule $\int \frac{P d x}{R}$, où $P$ est une fonction rationnelle de $x$, et $R$ la racine quarrée d'un polynome en $x$ du quatrième degré. Lagrange enfin est présenté d'un côté comme le continuateur d'Euler dont il prolonge et cherche à améliorer les résultats, et de l'autre comme l'auteur d'une méthode nouvelle permettant d'évaluer les intégrales elliptiques par des transformations successives, grâce à la comparaison de transcendantes successivement formées d'après la même loi, mais dont, malgré la conformité des résultats, Legendre marque toutefois la différence avec la méthode qui le conduit de son côté aux échelles de modules ${ }^{7}$. Bien qu'il n'établisse pas lui-même formellement de ligne de partage entre traditions de problèmes, du fait même qu'il cantonne Maclaurin et d'Alembert à un rôle mineur et qu'il mette en avant les travaux d'Euler qui se rapportent aux théorèmes d'addition, Legendre est amené à privilégier les contributions respectives de Landen et de Fagnano, lesquels apparaissent comme les initiateurs de points de vue différents selon que les intégrales elliptiques comparées sont, ou non, de modules différents $^{8}$. Sans doute, comme le rappelle Christian Gilain ([25, p. XXIX]), le rôle déterminant que l'historiographie des deux derniers siècles (par exemple [14], [36], [23], [35], [31], [32], [64], [9]) accorde à juste titre aux travaux d'Abel et de Jacobi et à l'inversion des intégrales elliptiques n'est pas étranger à cette évolution pour autant qu'il a conduit à accentuer davantage encore l'importance des recherches issues des travaux de Fagnano sur la mesure de la lemniscate et de leur reception par Euler en leur conférant le statut d'un courant indépendant.

Le tableau doit cependant être sensiblement nuancé et la présentation historique de Legendre mise en perspective. Dans l'étude qu'il consacre à l'histoire du problème de l'inversion des intégrales elliptiques ([37, p. 52-53]), comme dans l'avant-propos au volume 20 de la série I des Opera Omnia d'Euler ([38, p. VII-X]), Adolf Krazer distingue deux ensembles de mémoires

\footnotetext{
${ }^{5}$ Cf. [50, Avertissement, p. vi et vii], [50, Introduction, p. 2].

${ }^{6}$ Nous verrons plus loin que ce théorème joue un rôle important dans l'élaboration par Legendre de son propre résultat de rectification de l'hyperbole, et qu'il faudrait par conséquent en toute rigueur porter au crédit de Fagnano cette contribution à la tradition de problèmes liée à la transformation de Landen et à son utilisation dans la poursuite du projet principal de Legendre.

${ }^{7}$ Legendre se réfère au mémoire de Lagrange de 1785 , Sur une nouvelle méthode de calcul intégral ([39]). Notons à cet égard que la mention de cet aspect du travail de Lagrange disparaît entre l'édition de 1811 et celle de 1825 .

${ }^{8}$ En écho à ces remarques de Legendre, les recherches de Landen sur la rectification de l'hyperbole sont souvent présentées au XIXème siècle comme l'origine de la théorie de la transformation des intégrales elliptiques (cf. [57, Vorwort], [7, p. 6], [36, p. 2].), tandis qu'au siècle suivant certains auteurs voient dans les mémoires de Fagnano et Euler le commencement de la tradition de problèmes liée à la multiplication complexe (cf. [63, p. 10], [58, p. 249-51], [64, p. 305-6]).
} 
parmi ceux qu'Euler consacre aux intégrales elliptiques. Outre ceux qui s'inscrivent dans la suite de la réception par Euler des mémoires de Fagnano sur la lemniscate, Krazer attire en effet l'attention sur un deuxième groupe de mémoires qui s'inscrivent dans la filiation des recherches de Maclaurin et d'Alembert sur les méthodes d'intégration par arcs d'ellipse et d'hyperbole et dans lequels il repère le début de la réduction des intégrales elliptiques à des formes normales. En reprenant ces analyses, Christian Gilain ([24, p. XL]) montre en outre, à la lumière de la correspondance entre les deux hommes, qu'Euler estimait fort les recherches de d'Alembert sur ce sujet et qu'il y eut entre eux un échange de vues portant sur la comparaison de leurs méthodes respectives.

Il est donc particulièrement significatif que, dans ses présentations historiques [cf. Appendice], Legendre ne mentionne que les mémoires d'Euler qui appartiennent au premier groupe distingué par Krazer, à savoir ceux qui sont publiés dans les tomes 6 et 7 de l'Académie de Saint-Petersbourg (parmi lesquels notamment [15], [16] et [17]), et non ceux du second groupe, publiés dans les tomes 8 et 10, alors que, comme nous le verrons plus loin, certains autres passages suggèrent qu'il en avait connaissance, comme semble en témoigner son allusion à peine voilée au mémoire ([18]) dans lequel Euler propose d'introduire des symboles spécifiques pour les arcs d'ellipse, considérés comme de nouvelles transcendantes au même titre que les logarithmes et les fonctions trigonométriques. En occultant ainsi la filiation de Maclaurin et d'Alembert à Euler, Legendre conforte de fait une opposition construite entre les recherches de Landen sur la rectification de l'hyperbole et celles de Fagnano sur la mesure de la lemniscate. En choisissant en effet de faire du résultat de Landen et de son appropriation un moment instaurateur dans la poursuite de ses fins propres, il contribue du même coup à préciser la définition de son projet mathématique. Cette forme de réflexivité laisse alors supposer que l'identification de ce qui dans la perspective de Legendre devient la "transformation de Landen" dépend de conditions qu'il faut analyser précisément. En qualifiant le théorème de Landen de "découverte mémorable qui simplifie la théorie des transcendantes, et qui aurait pu conduire l'auteur à d'autres résultats plus importans" 9 , Legendre suggère en effet en demi-teintes que Landen ne voyait sans doute pas dans la rectification de l'hyperbole ce que lui-même y discernait.

S'agissant de la transformation de Landen, cette question des conditions d'identification a fait l'objet d'évaluations contrastées. Plusieurs problèmes ont en effet été soulevés concernant les relations entre les trois principaux protagonistes auxquels on pourrait prétendre imputer la découverte de cette transformation, à savoir Landen, Lagrange et Legendre. Bien qu'il interprète immédiatement le théorème géométrique de Landen comme une transformation entre intégrales elliptiques ${ }^{10}$, Richelot (1868) reconnaît toutefois n'avoir pas été en mesure de déterminer si Landen avait déjà lui-même remarqué, dans les trois sections coniques de son théorème de rectification, la propriété que Lagrange le premier aurait par la suite approfondie sous la forme d'une transformation analytique dans l'algorithme de la moyenne arithmético-géométrique ${ }^{11}$. Aucun élément de réponse n'est alors fourni pour expliquer ce qui du même coup se présenterait comme un double silence, de Lagrange sur Landen et de Legendre sur Lagrange. Richelot se contente de souligner que le fait, réel ou supposé, que Lagrange ne mentionne nulle part Landen devrait paraître "moins frappant" [weniger auffallend] que le fait que Legendre ne mentionne

\footnotetext{
${ }^{9}[50$, p. 2].

${ }^{10}$ Dans un appendice de son livre ([57, p. 55-6]), Richelot (1868) expose brièvement la démarche géométrique de Landen, puis partant de la transformation analytique, en propose une interprétation géométrique, analogue à celle de Jacobi ([33, p. 178-179]), en remodelant la configuration géométrique des trois sections coniques afin que les moyennes arithmétique et géométrique y apparaissent plus clairement.

${ }^{11}[57$, p. 57] : "Ich habe nicht ermitteln können, ob Landen selbst schon, eine sich fast von selbst darbietende Eigenschaft der drei von ihm betrachteten Kegelschnitte bemerkt hat, welche zuerst von Lagrange in der Abhandlung in den Turiner Memoiren 1784-85, pag. 237 in dem Algorithmus des arithmetisch-geometrischen Mittels bei dieser Transformation weiter verfolgt worden ist".
} 
pas non plus le mémoire de Lagrange lorsqu'il est question de la découverte de la transformation de Landen, même s'il établit un lien entre la transformation de Lagrange et celle de Landen ${ }^{12}$. Comme Richelot avant lui, Enneper (1876/1890) déplore que, dans son Traité de 1825, Legendre ait minimisé l'apport de Lagrange en ne mentionnant les recherches de son prédécesseur qu' "en passant" [nur sehr vorübergehend] et dans des termes qui ne rendent pas justice à la richesse de leur contenu ${ }^{13}$. Mais Enneper va plus loin que Richelot en supposant, à tort comme nous le verrons, que Lagrange ne connaissait pas le théorème de Landen, ce qu'il croit pouvoir inférer du fait que le mathématicien français estimait sa méthode "d'un genre assez nouveau" pour qu'il soit utile de "l'appliquer en détail à la rectification des arcs elliptiques et hyperboliques"14. Si pour Casorati (1868), la transformation de Lagrange n'est rien d'autre que la transformation donnée par Landen "sous une autre forme" ${ }^{15}$, Mansion (1870) de son côté n'hésite pas à les déclarer identiques ${ }^{16}$. Koenigsberger (1879) en revanche souligne le statut ambivalent des résultats de Landen et d'autres géomètres de cette période, pour autant qu'ils dépendent d'un côté des considérations géométriques portant sur les arcs de sections coniques ou d'autres courbes algébriques, qui ont permis de les obtenir, mais fournissent d'un autre côté des relations et des formules de réduction entre intégrales elliptiques. Sur la base d'une telle différenciation entre le résultat géométrique et sa formulation analytique, Koenigsberger considère qu'en dégageant la transformation proprement dite, Legendre montre non seulement qu'on peut retrouver les propositions géométriques de Landen à partir de la relation analytique entre intégrales elliptiques, mais aussi que le principe de la transformation est beaucoup "plus important" [weit wichtiger] que toutes les conséquences géométriques qu'on peut en tirer, parce qu'il permet d'accéder à un "point de vue analytique" ouvrant la voie aux échelles de modules ${ }^{17}$. Fricke (1913) annule par la suite toute forme de hiérarchisation entre résultat géométrique et formulation analytique, en imputant à Landen une appréhension directe de la transformation analytique dont il aurait simplement déduit le résultat géométrique relatif à la rectification de l'hyperbole ${ }^{18}$, là où Lagrange n'aurait fait que retrouver "indépendamment" cette même transformation et Legendre ne se serait illustré qu'en la "mettant à nouveau en relief" [aufs neue hervorgehoben $]^{19}$.

Pour tenter de répondre aux questions ainsi laissées en suspens, nous nous proposons ici de retracer la préhistoire de la transformation de Landen des premières recherches sur la rectification des courbes par arcs de sections coniques au début du XVIIIème siècle à la constitution du damier analytique dans l'œuvre de la maturité de Legendre, en nous attachant aux formes concrètes d'appropriation et de réélaboration des contenus mathématiques en fonction des contextes de réception spécifiques. Nous montrerons d'abord comment le théorème de rectification de Landen

\footnotetext{
${ }^{12}[57$, Vorwort] : "Dass Lagrange in seiner Abhandlung (Turiner Memoiren 1784, p. 218) worin er die später nach Gauss genannte Transformation der elliptischen Integrale und den Algorithmus der arithmetisch geometrischen Mittel angegeben hat, nirgend der Arbeit Landens erwähnt, dürfte weniger auffallend sein, als dass Legendre nirgend der Abhandlung von Lagrange erwähnt, obgleich er den Zusammenhang der Lagrange'schen und Landen'schen Transformations-Formeln (Théorie des f. e., p. 89) angiebt."

${ }^{13}[14, \S 44$, p. 357] : "In seinem grossen "Traité" erwähnt Legendre der tiefen und klaren Untersuchungen des grossen Mathematikers nur sehr vorübergehend und in Worten, welche mit dem reichen Inhalte der Arbeit von Lagrange in keinem rechten Verhältnis stehen".

$14[39$, p. 283]

$15[7$, p. 6-7].

16 [54, p. 16] : "la substitution de Lagrange est identique à celle de Landen."

17 [36, p. 9] : "[Legendre] zeigt, dass sich aus dieser einfachen analytischen Beziehung die Sätze von Landen, wonach sich ein Hyperbelbogen durch zwei Ellipsenbögen ausdrücken lässt etc., unmittelbar ergeben, dass aber weit wichtiger als alle dieser geometrischen Folgerungen das in der erwähnten Substitution liegende Transformationsprincip sei, wonach durch wiederholte Anwendung dieser Substitution eine Kette von unendlich vielen Moduln hergestellt werden kann, welche Veranlassung geben zu einfachen Methoden für die Berechnung der vollständigen und unvollständigen elliptischen Integrale erster Gattung, ..."

${ }^{18}[23$, p. 190-1] : "Er benutzte seine Transformation zur Herleitung des nach ihm benannten Theorems, daß der Bogen einer gleichseitigen Hyperbel sich durch die Differenz zweier Ellipsenbögen ausdrücken lasse."

${ }^{19}[23$, p. 191].
} 
s'inscrit dans la filiation de recherches antérieures de Maclaurin et d'Alembert, en mettant en lumière les raisons qui conduisent à penser que la transformation analytique n'est encore qu'implicite dans la propriété des trois coniques $(\S 2)$. Nous analyserons ensuite précisément les deux mémoires de Legendre, Sur les intégrations par arcs d'ellipse (1786). Nous montrerons comment ce dernier s'approprie par étapes le résultat de Landen en le déduisant d'un théorème analogue sur la réduction de l'arc d'hyperbole aux arcs d'ellipse, qu'il avait d'abord obtenu, dans le premier mémoire, indépendamment de Landen, par une méthode fondée sur la variation continue des paramètres des intégrales elliptiques. En comparant les deux démonstrations, nous établirons ainsi que la lecture originale que Legendre propose du résultat de Landen permet de dégager la transformation analytique proprement dite de son vêtement géométrique (§3). Enfin, dans un dernier temps, en montrant comment la transposition aux fonctions de première espèce de ces mêmes méthodes d'approximation élaborées dans le cadre restreint d'un calcul des arcs d'ellipse, conduit à la théorie du damier analytique, nous chercherons à cerner la spécificité de ces méthodes par rapport à celle de Lagrange $(\S 4)$.

\section{Le théorème de Landen}

La connaissance que nous avons des travaux mathématiques de John Landen (1719-90), mathématicien anglais ayant exercé les fonctions d'arpenteur-géomètre pendant la plus grande partie de sa vie ${ }^{20}$, s'est précisée à mesure que l'historiographie s'écartait de l'image d'un simple précurseur de Legendre ayant fait "une découverte géométrique tout à fait inattendue" ${ }^{21}$ (Montucla 1802), ou encore "ayant eu la bonne fortune de tomber sur une remarque inattendue qui a formé le point de départ de la théorie des fonctions elliptiques, à laquelle son nom restera attaché" 22 (Marie 1886). Le théorème de Landen sur la réduction de l'arc hyperbolique à deux arcs elliptiques assignables apparaissait ainsi comme "une vérité singulière (...) ensuite démontrée plus simplement par Legendre, $[$ car, ajoute Montucla $]$ on sait assez que rarement les premiers inventeurs ont pris le chemin le plus court" ${ }^{23}$. Outre la rectification de l'hyperbole, Montucla présente Landen comme "l'inventeur d'une analyse particulière, qu'il nomme résiduelle, et dont il publia en 1755 [sic] une annonce et un essai" ${ }^{24}$. Dans une deuxième phase de l'historiographie, les travaux de Florian Cajori à la toute fin du XIXème siècle et au début du siècle suivant ont brossé un tableau plus équilibré de l'œuvre. Quoiqu'il présente toujours la rectification de l'ellipse comme la "découverte capitale" ${ }^{25}$ de Landen, Cajori étudie de manière plus approfondie l' "analyse résiduelle" par laquelle le mathématicien anglais "a tenté d'éviter les difficultés métaphysiques liées aux fluxions en adoptant une méthode purement algébrique" ${ }^{26}$. Plus récemment, en se démarquant de certains partis pris de Cajori ${ }^{27}$, le livre de Niccolò Guicciardini a permis de mieux cerner la place qui revient à Landen dans le courant des "fluxionistes analytiques", lesquels se sont efforcés dans les années 1755-1785 d'élaborer un calcul qui ne soit pas

\footnotetext{
${ }^{20}$ Sur la vie et l'œuvre de John Landen, cf. [30]. Les éléments biographiques le concernant semblent avoir connu certaines fluctuations puisque Montucla assez étrangement le fait mourir "en 1776 ou 1777" ([56, p. 240]).

${ }^{21}[56$, p. 240$]$

$22[55$, p. 243$]$.

$23[56$, p. 240$]$

${ }^{24}[56$, p. 240]. Montucla semble ici se référer au livre Mathematical Lucubrations de 1755 ([41]), là où on attendrait plutôt le Discourse concerning residual analysis de 1758 ([42]) et The residual analysis de 1764 ([43]).

$25[4$, p. 247]

$26[4$, p. 247].

${ }^{27}$ Cajori aborde la contribution de Landen au chapitre IX, "Abortive attempts at arithmetisation", de son livre sur l'histoire des notions de limite et de fluxion en Grande-Bretagne au XVIIIème siècle ([5]). Plus généralement, il envisage cette période des mathématiques britanniques principalement comme une période de stagnation ou d'essais avortés. Niccolò Guicciardini au contraire s'assigne la tâche de mettre en lumière ce que les mathématiciens anglais ont réalisé sans recourir à une telle grille de lecture préalable.
} 
immédiatement interprétable en termes géométriques ou cinématiques ${ }^{28}$. Le but de la Residual Analysis est ainsi d'établir le calcul sur la base des "principes anciennement-reçus de l'algèbre" et non sur ceux "empruntés à la doctrine du mouvement" ${ }^{29}$, sans par conséquent recourir ni aux fluxions ni aux différentielles. Pour faire comprendre le sens de cette démarche, Landen donne, dès les premières pages de son Discourse de 1758, un exemple caractéristique que la plupart des commentateurs commentent à juste titre en détail ${ }^{30}$.

In the application of the Residual Analysis, a geometrical or physical problem is naturally reduced to another purely algebraic; and the solution is then readily obtained, without any supposition of motion, and without considering quantities of as composed of infinitely small particles.

It is by means of the following theorem, viz.

$$
\frac{x^{\frac{m}{n}}-v^{\frac{m}{n}}}{x-v}=x^{\frac{m}{n}-1} \times \frac{1+\frac{v}{x}+\left(\frac{v}{x}\right)^{2}+\left(\frac{v}{x}\right)^{3}}{1+\left(\frac{v}{x}\right)^{\frac{m}{n}}+\left(\frac{v}{x}\right)^{\frac{2 m}{n}}+\left(\frac{v}{x}\right)^{\frac{3 m}{n}}}\left(\frac{(m)}{(n)}\right.
$$

(where $m$ and $n$ are integers) that we are enabled to perform all the principal operations in our said Analysis; and I am not a little surprized, that a theorem so obvious, and of such vast use, should so long escape the notice of algebraists. ${ }^{31}$

Cette identité algébrique s'obtient en effet immédiatement par la reformulation des séries géométriques du membre de droite. Pour différentier $x^{\frac{m}{n}}$, il suffit alors de prendre $x=v$ de sorte que le rapport des séries donne le coefficient rationnel $\frac{m}{n}$. Comme le note Niccolò Guicciardini, cette procédure qui ne vaut que pour un exposant rationnel supposait une conception de l'égalité algébrique selon laquelle l'indétermination qui peut résulter de certaines substitutions n'est imputable qu'à la forme particulière de l'expression et n'affecte pas la vérité universelle de l'équation (ici la rapport des séries permet de lever l'hypothèque que représente le rapport indéterminé $\frac{0}{0}$ lorsque l'on prend $x=v$ ). Landen souligne que sa méthode permet, contrairement à ce que font ordinairement les fluxionistes, d'éviter de recourir au théorème du binôme, "which is much more difficult to investigate than the limit they are seeking" 32 .

Niccolò Guicciardini remarque que Landen n'a pas utilisé dans ses autres travaux la technique algébrique développée dans The Residual Analysis, et qu'en particulier il recourt explicitement à une méthode des limites dans les investigations qui relèvent du calcul intégral. Si Landen utilise en effet la notation et la terminologie des fluxions dans ses recherches sur l'intégration de certaines intégrales [nommées par lui "fluentes"] par arcs de sections coniques, et si, qui plus est, comme nous le verrons plus bas, il démontre son théorème sur la rectification de l'hyperbole en utilisant un lemme de Maclaurin qui requiert la considération de triangles infinitésimaux, il n'en reste pas moins que le ressort principal de la méthode qui lui permet d'obtenir ce résultat est là aussi foncièrement algébrique. La réduction de l'arc hyperbolique à deux arcs elliptiques résulte en effet de la décomposition algébrique d'une expression rationnelle en une somme de deux expressions rationnelles plus simples, en sorte que la procédure algébrique opère sur les termes fluxionnels en mettant de côté ce que Landen nomme leur "figurative sense"33. Cette importance donnée à la préparation algébrique dans l'élaboration des problèmes de géométrie constitue précisément l'un des traits que G. N. Watson devait plus tard souligner, pour le

\footnotetext{
${ }^{28}$ Niccolo Guicciardini montre ainsi que John Landen fait partie d'un courant qui comprend aussi Thomas Simpson (1710-61) et Edward Waring (1736-98), cf. [29, ch. 6].

${ }^{29}[42$, p. 4$]$.

${ }^{30}$ Cf. [8, p. 583], [5, p. 233], [29, p. 86].

31 [42, p. 5].

$32[42$, p. 9$]$.

$33[42$, p. 4$]$.
} 
stigmatiser et justifier ainsi la préférence accordée à la présentation que Legendre propose du résultat de Landen ${ }^{34}$.

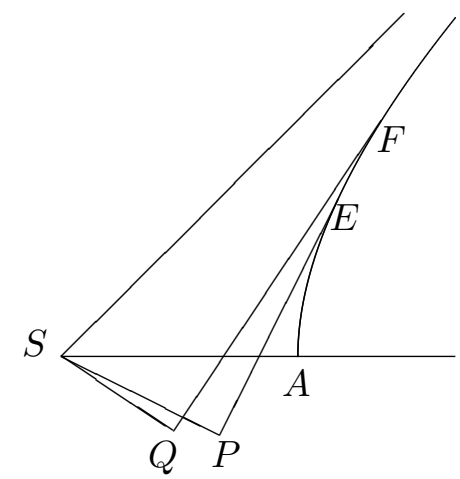

Fig. 1 - L'excès de la tangente sur l'arc d'hyperbole

Landen présente ses recherches sur la réduction des fluentes aux arcs de sections coniques et la rectification de l'hyperbole dans deux mémoires ([44], [45]) publiés dans les Philosophical Transactions en 1771 et en 1775 . Dans le premier mémoire, il se réfère explicitement aux méthodes d'intégration par arcs hyperboliques et elliptiques développées par Maclaurin et d'Alembert, et qui utilisent comme l'une de leurs chevilles ouvrières, la différence entre l'arc d'hyperbole et sa tangente (cf. Fig. 1). Toutefois cette différence n'étant en toute rigueur déterminée que pour des valeurs finies de l'arc et de la tangente, les théorèmes qui en dépendent n'ont pas la généralité qu'on en attendrait. Le but que Landen se fixe consiste donc à trouver une expression adéquate pour ces théorèmes qui permette d'en assigner la limite lorsque l'arc et la tangente deviennent infinis. À la fin de son premier mémoire, Landen annonce la démonstration de la rectification de l'hyperbole qu'il donne quelques années plus tard dans le second mémoire.

\subsection{Maclaurin et d'Alembert}

Dans son Treatise of fluxions (1742, Book II, chap. III, art. 755) ${ }^{35}$, Maclaurin définit un programme de recherches dans la lignée duquel s'inscrivent les travaux de d'Alembert sur les différentielles qui se réduisent à la rectification de l'ellipse ou de l'hyperbole. Maclaurin développe une conception du calcul intégral fondée sur la classification des fluentes selon une hiérarchie qui se justifie, chemin faisant, par l'irréductibilité empiriquement constatée des classes d'intégrales ainsi définies. Il distingue en effet plusieurs degrés de résolution selon que la fluente peut être assignée en un nombre fini de termes algébriques (c'est le cas par exemple de la fluente de $\frac{\dot{x}}{\sqrt{1 \pm x}}$ ), qu'elle s'exprime au moyen de logarithmes ou d'arcs de cercles (la fluente de $\frac{\dot{x}}{\sqrt{1 \pm x^{2}}}$ ), ou enfin qu'elle peut être ramenée à des arcs de sections coniques (les fluentes de $\frac{\dot{x} \sqrt{x}}{\sqrt{1 \pm x^{2}}}$ et de $\left.\frac{\dot{x}}{\sqrt{x} \sqrt{1 \pm x}}\right)$. S'il est assez naturel de passer du premier échelon au deuxième par adjonction aux termes algébriques des transcendantes usuelles en Analyse, logarithmes et arcs de cercles, et du deuxième échelon au troisième en recevant aussi les transcendantes qui apparaissent comme les

\footnotetext{
${ }^{34}$ Cf. [63, p. 13] : "I take ... a very beautiful theorem, first discovered in an algebraic form by Fagnano, given a geometrical interpretation by Euler, and then modified and developed by Landen. The investigation which I give is due to Legendre; it seems to me much more elegant than the earlier work on the theorem. (I remark in parenthesis that it has been my experience ...that the technique of eighteenth century mathematicians in dealing with problems of pure calculus was considerably superior to their technique in analytical geometry, and that frequently the algebraical work which they found necessary in solving problems of analytical geometry can only be described as clumsy when compared with their other work)".

${ }^{35} \mathrm{Cf}$. le commentaire de Christian Gilain dans ([24, p. 219-220], [25, p. XXXII]).
} 
plus simples après les logarithmes et les arcs de cercle, notons toutefois que Maclaurin réorganise ces échelons d'une manière un peu différente dans un autre passage du traité en les apparentant deux par deux selon leur caractère de quadrature ou de rectification.

art. 798. After the fluents that can be accurately assigned in finite terms by common algebraic expressions, and those which can be reduced to circular arks and logarithms, the fluents that deserve the next place are such as are assigned by hyperbolic and elliptic arks; which with the former are all comprehended under these which are measured by the lines that bound the conic sections (the triangle and circle being figures of this kind), as the first two are measured by the areas of conic sections. ${ }^{36}$

Dans la seconde partie de son mémoire de 1746 portant sur les différentielles qui se rapportent à la rectification de l'ellipse ou de l'hyperbole, d'Alembert se propose de continuer les recherches de Maclaurin, " $\&$ de les pousser plus loin" 37. Mais comme l'a montré Christian Gilain, si d'Alembert s'inscrit dans la lignée des travaux du mathématicien écossais dont il récapitule les principaux résultats selon des principes d'exposition différents, il s'en démarque toutefois nettement par les méthodes employées. Là où, chez Maclaurin, l'intégration des différentielles irrationnelles par arcs de sections coniques se faisait au moyen de raisonnements géométriques plutôt que par calculs algébriques, d'Alembert privilégie au contraire une "démarche systématiquement analytique" en ceci qu' "il ne construit aucune figure mais développe des calculs algébriques sur les différentielles et sur leurs intégrales, à l'aide essentiellement de changements de variables, ces transformations étant liées aux propriétés algébriques de la différentiation des fonctions et donc à celle de l'intégration indéfinie considérée comme opération inverse de la différentiation" 38 . D'Alembert étudie ainsi de manière systématique les intégrales de la forme $\int \frac{d x}{\sqrt{x} \sqrt{P}}$ et $\int \frac{\sqrt{x} d x}{\sqrt{P}}$ où $P$ est un polynôme quadratique ${ }^{39}$, et en distinguant différents cas selon la forme du polynôme $P$, montre qu'elles se réduisent toutes par ce type de transformations algébriques à des arcs d'ellipse et d'hyperbole. Il considère ainsi douze "problèmes" qu'il ordonne par complexité croissante de sorte que les plus composés se ramènent aux plus simples et tous finalement aux deux "lemmes" fondamentaux qui établissent la forme générale des intégrales indéfinies qui représentent les longueurs d'arcs de l'ellipse et de l'hyperbole

$$
\begin{gathered}
\int \frac{d x \sqrt{x}}{\sqrt{P}} \text { avec } P=f x-x^{2}-g^{2} \quad \text { (ellipse) } \\
\int \frac{d x \sqrt{x}}{\sqrt{P}} \text { avec } P=x^{2}-g^{2} \pm f x \quad \text { (hyperbole). }
\end{gathered}
$$

Pour obtenir son théorème sur la rectification de l'hyperbole au moyen de deux ellipses, Landen utilise ainsi un résultat de Maclaurin établissant dans le cas particulier de l'hyperbole équilatère la fluente correspondant à l'excès de la tangente sur l'arc d'hyperbole (cf. Fig. 1),

$$
E P-\operatorname{arc} A E=\int_{1}^{z} \frac{-\sqrt{z} d z}{2 \sqrt{1-z^{2}}}
$$

mais sous une forme plus générale qu'il emprunte selon toute vraisemblance à la présentation algébrique de d'Alembert, bien différente de la démonstration de caractère géométrique qu'en donne Maclaurin au chapitre III du second livre du Treatise of fluxions.

\footnotetext{
${ }^{36}[53$, Book II, chap. III, art. 798].

$37[11$, p. $200(129)]$.

38 [25, p. XXXIII]. Cf. aussi [24, p. 220-221].

${ }^{39}$ Les deux types d'intégrales considérées ici par d'Alembert sont des intégrales elliptiques respectivement de première et de seconde espèce. Sur la correspondance entre les intégrales de d'Alembert et les formes canoniques de Legendre, cf. [25, p. XXXV].
} 
art. 799. Let $A E H$ be an equilateral hyperbola, that has its centre in $S$ and vertex in $A$, $A D$ a right line perpendicular to $S A$, suppose $S A=1, S N=x$, and let a circle described with the radius $S N$ from the centre $S$ meet $A D$ in $M$, let $S E$ bisect the angle $A S M$, and meet the hyperbola in $E$; then the hyperbolic ark $A E$ shall be equal to the fluent of $\frac{\dot{x} \sqrt{x}}{2 \sqrt{x x-1}}$. For let the ark $A E=s, S E=r$, and $S P$ be perpendicular on $E P$ the tangent of the hyperbola in $P$; then the triangles $S M A$ and $S E P$ will be similar, by art. 181, and $\dot{s}: \dot{r}:: S E: E P$ :: $S M: A M:: x: \sqrt{x x-1}$; but $S A, S E$ and $S M$ are in continued proportion, or $r=\sqrt{x}$, so that $\dot{r}: \dot{x}:: 1: 2 \sqrt{x}$; consequently $\dot{s}=\frac{\dot{x} \sqrt{x}}{2 \sqrt{x x-1}}$; and supposing the fluent of $\frac{\dot{x} \sqrt{x}}{\sqrt{x x-1}}$ to begin to be generated when $x=1$, and thereafter to increase while $x$ increases, it will be always equal to $2 A E$. If $A m$ be perpendicular to $S M$ in $m$, and we now suppose $S m=x$, then the hyperbolic ark $A E$ will be the fluent of $\frac{-\dot{x}}{2 x \sqrt{x} \sqrt{1-x x}}$ (as will appear by substituting in the former fluxion $x^{-1}$ for $\left.x\right)$; and $E P-A E$ the excess of the tangent above the hyperbolic ark $A E$ will be the fluent of $\frac{-\dot{x} \sqrt{x}}{2 \sqrt{1-x x}}$; because $E P$ will then be equal to $\sqrt{\frac{1}{x}-x}$, and its fluxion to $\frac{-\dot{x}-x x \dot{x}}{2 x \sqrt{x} \sqrt{1-x x}} \cdot 40$

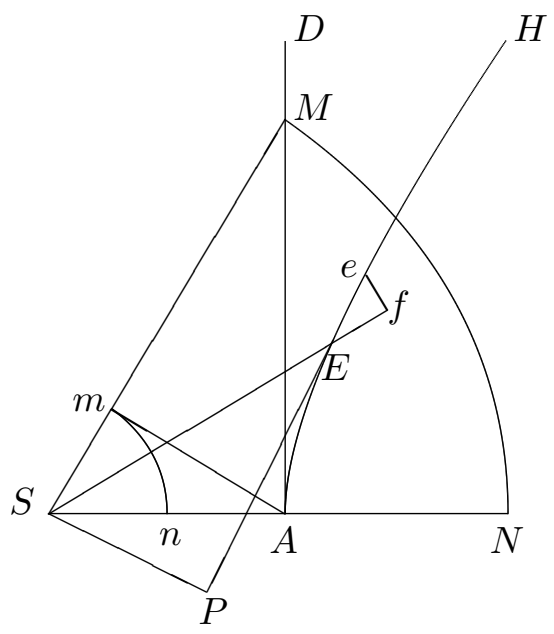

Fig. 2 - La démonstration de Maclaurin

La démonstration de Maclaurin met en jeu trois composantes principales : (1) une propriété liant la fluxion d'arc à la fluxion du rayon à la courbe issu d'un point fixe, (2) un ensemble de relations de similitude liant deux à deux quatre triangles de la figure dont un triangle infinitésimal, enfin (3) un changement de variable qui se présente sous une forme géométrique comme la mise en corrélation des rayons de deux cercles permettant de construire l'hyperbole par points. La première propriété liant fluxion de l'arc et fluxion du rayon est acquise dès 1720 . Dans un mémoire publié dans les Philosophical Transactions ([52]), le théorème est énoncé sous la forme d'une proportion : la fluxion de l'arc est à la fluxion du rayon comme le rayon est à la tangente et s'obtient en considérant deux points très proches l'un de l'autre, soient $L$ et $l$, sur une courbe $B L l$ (cf. Fig. 3, partie gauche). En prenant $S L$ le rayon issu d'un point $S$ arbitrairement choisi, $L P$ la tangente à la courbe en $L$, lo la perpendiculaire à $L S$ issue de $l$, on peut interpréter $L l$ comme la fluxion de l'arc $B L$, et $L o$ comme la fluxion du rayon. La similitude des triangles $L l o$ et $L S P$ donne alors la proportion souhaitée

$$
\frac{L l}{L o}=\frac{S L}{P L} .
$$

Dans le Treatise of fluxions, Livre I, chap. VII, "Of tangents", art. 206, Maclaurin exprime la même proposition sous une forme légèrement différente (cf. Fig. 3, partie droite) : "the fluxion

\footnotetext{
${ }^{40}$ [53, Book II, chap. III, art. 799]
} 
of the curve $F E$ shall be to the fluxion of the ray $S E$ as the tangent $E D$ is to the ray $S E$ ". Les deux formulations sont parfaitement équivalentes et correspondent à des manières différentes d'assigner la tangente. Dès le mémoire de 1720, Maclaurin développe à l'aide de cette proposition, une méthode, plus tard appelée méthode des podaires, qui permet d'engendrer une courbe à partir d'une courbe donnée en prenant le lieu géométrique des points où les perpendiculaires abaissées du centre rencontrent les tangentes à la courbe.
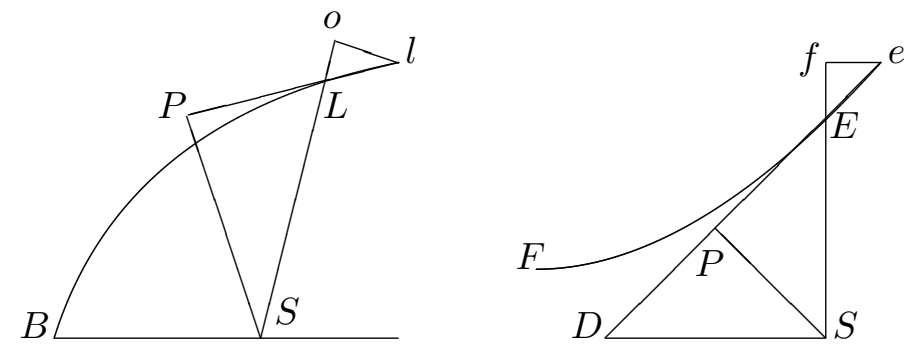

FIG. 3 - La propriété liant la fluxion de l'arc à la fluxion du rayon

Dans le cas de l'hyperbole équilatère (cf. Fig. 2), en prenant l'arc $A E=s$ et le rayon $S E=r$ à partir du centre $S$, la proposition précédente de Maclaurin donne d'abord immédiatement la similitude des triangles $S P E$ et efE. Comme d'autre part les triangles $S M A$ et $S E P$ sont semblables, on a en outre les proportions suivantes

$$
\frac{d s}{d r}=\frac{S E}{E P} \quad \text { et } \quad \frac{S E}{E P}=\frac{S M}{A M},
$$

d'où l'on tire, puisque $A M=\sqrt{x^{2}-1}$ par application du théorème de Pythagore au triangle $A M S$,

$$
\frac{d s}{d r}=\frac{x}{\sqrt{x^{2}-1}} .
$$

Maclaurin fait la substitution que suggère la remarque que les segments $S A, S E$ et $S M$ sont en proportion continue ${ }^{41}$, c'est-à-dire $r=\sqrt{x}$, par suite $d r=\frac{d x}{2 \sqrt{x}}$, d'où il déduit la valeur de l'élément d'arc

$$
d s=\frac{\sqrt{x} d x}{2 \sqrt{x^{2}-1}},
$$

où $x$ croît à partir de la valeur 1 , à mesure que le point $N$ s'éloigne de $A$ le long du grand axe.

Dans une deuxième étape de la démonstration, Maclaurin opère un changement de variable en posant $z=\frac{1}{x}$, où $z$ est compris entre 0 et 1 , de manière à obtenir une nouvelle expression pour l'élément d'arc

$$
d s=\frac{-d z}{2 z \sqrt{z} \sqrt{1-z^{2}}}
$$

qu'on intègre de 1 à $z$ pour obtenir la valeur de l'arc $A E$. Ce changement de variable correspond à la construction géométrique qui consiste à abaisser la perpendiculaire issue de $A$ à $S M$ de façon à obtenir un point $m$ qui permet de déterminer $S m=S n=z$. On montre aisément que les rayons $S n$ et $S N$ des deux cercles sont inverses l'un de l'autre ${ }^{42}$. On remarque qu'on peut

\footnotetext{
${ }^{41}$ En prenant les coordonnées polaires $(\xi, \eta)$ du point $E$ sur l'hyperbole équilatère $\xi^{2}-\eta^{2}=1$, on obtient la relation $r^{2} \cos ^{2} \phi-r^{2} \sin ^{2} \phi=1$, d'où l'on tire $r^{2} \cos 2 \phi=1$. Mais par construction, l'angle $A S M$ est le double de l'angle $A S E$, par conséquent $\cos 2 \phi=\frac{A S}{S M}=\frac{1}{x}$.

${ }^{42}$ Les deux triangles $S m A$ et $S A M$ sont en effet semblables.
} 
aussi bien construire l'hyperbole par points en partant du segment $S n$ que du segment $S N$, et que le changement de variable n'induit qu'une construction différente ${ }^{43}$.

En exprimant la tangente $E P$ en fonction de la nouvelle variable $z=S m$, Maclaurin obtient enfin la formule ${ }^{44} E P=\sqrt{\frac{1}{z}-z}$, qu'il exprime comme une fluente en intégrant la fluxion correspondante, ce qui lui permet de retrouver, pour la tangente $E P$, une fluxion, ou une différentielle, irrationnelle de même forme que pour l'arc $A E$

$$
E P=\int_{1}^{z} \frac{\left(-1-z^{2}\right) d z}{2 z \sqrt{z} \sqrt{1-z^{2}}}
$$

d'où résulte l'intégrale qui exprime l'excès de la tangente $E P$ sur l'arc $A E, \int_{1}^{z} \frac{-\sqrt{z} d z}{2 \sqrt{1-z^{2}}}$.

Le résultat de Maclaurin sera repris sous une forme plus générale dans le premier des douze problèmes de d'Alembert ${ }^{45}$ portant sur la différentielle irrationnelle

$$
\int \frac{d x \sqrt{x}}{\sqrt{P}} \text { avec } P=b^{2} \pm f x-x^{2},
$$

dont le mathématicien français montre qu'elle peut se réduire à la somme d'un arc d'hyperbole de semi-axes $b$ et $\pm \frac{f}{2}+\sqrt{\frac{f^{2}}{4}+b^{2}}$ et d'un terme algébrique correspondant à la tangente. La méthode algébrique par laquelle d'Alembert se démarque de Maclaurin, auquel il reprochait de n'avoir "pas même donné l'Analyse" 46 de ses résultats ou de n'avoir employé dans la réduction de ses fluentes qu' "une espèce de synthèse sans montrer la route qu'il a suivie pour y parvenir" 47 , permet ainsi d'étendre à des paramètres arbitraires la proposition que son prédécesseur n'avait démontré que dans le cas particulier de l'hyperbole équilatère. Bien que, comme le souligne Christian Gilain ${ }^{48}$, d'Alembert n'indique pas la méthode utilisée pour trouver ce résultat, il semble qu'en l'occurrence il ait en effet fourni, conformément à ce qu'il avait annoncé, l'analyse sous-jacente qui manquait à la "synthèse" géométrique proposée par Maclaurin. En adoptant une démarche inverse à celle du mathématicien écossais, il part de la différentielle irrationnelle $\int \frac{d x \sqrt{x}}{\sqrt{b^{2} \pm f x-x^{2}}}$ au lieu d'y aboutir, et opère, mais cette fois-ci dans le sens inverse, le même changement de variable, à la généralité des paramètres près (en l'occurrence $z=\frac{b^{2}}{u}$ ), de façon à obtenir l'expression qui, à la faveur d'une intégration par parties, fasse apparaître l'arc et la tangente.

\subsection{La rectification de l'hyperbole au moyen de deux ellipses}

Dans l'article I de son premier mémoire de 1771, Landen reprend, sans démonstration, avec pour toute justification les mots "as is well known" ${ }^{49}$, la proposition générale de d'Alembert, quoiqu'à un changement de variable près, de façon à l'ajuster au cas d'une hyperbole de semi-axe transverse $m$ et de semi-axe conjugué $n$. L'intégrale exprimant l'excès de la tangente sur l'arc

\footnotetext{
${ }^{43}$ Supposons que le point $n$ parcoure le segment $A S$ de $A$ vers $S$ de sorte que $S n=z$ varie de 1 à 0 . Pour chaque position de $n$, on trace le cercle de centre $S$ et de rayon $S n$, puis on trace la tangente issue de $A$ à ce cercle en un point $m$, laquelle est donc perpendiculaire au rayon $S m$. Il suffit alors de tracer la bissectrice de l'angle $A S m$ et de la prolonger jusqu'à l'hyperbole en $E$.

${ }^{44}$ Les triangles $A m S$ et $M A S$ d'une part et $M A S$ et $E P S$ d'autre part étant semblables, on a par conséquent $\frac{E P}{S E}=\frac{A m}{S A}$, où $S A=1, S E=r=\frac{1}{\sqrt{z}}$ et $A m=\sqrt{1-z^{2}}$, d'où l'on tire l'expression souhaitée pour $E P$.

${ }^{45}$ Cf. [11, p. 203 (133-4)].

$46[11$, p. $200(129)]$

${ }^{47}[11$, p. 205 (135)]

${ }^{48}[25$, note 114, p. 134$]$.

${ }^{49}$ [44, p. 299].
} 
d'hyperbole, donnée en 1771 sous la forme ${ }^{50}$

$$
E P-\operatorname{arc} A E=\int_{m}^{z} \frac{-\frac{1}{2} \sqrt{m} \sqrt{z} d z}{\sqrt{n^{2}+2 f z-z^{2}}}
$$

où $f=\frac{m^{2}-n^{2}}{2 m}$, constitue alors l'une des pierres angulaires du théorème de rectification dont la démonstration ne sera donnée que dans le mémoire de 1775.

Dans ce second mémoire, Landen déduit son théorème de rectification de trois lemmes géométriques dont les deux premiers fournissent des intégrales apparentées par leur forme, que l'équation algébrique donnée par le troisième lemme permet de mettre en relation (cf. Fig. 4).

\section{hyperbole}

semi-axe transverse : $m-n$ semi-axe conjugué : $2 \sqrt{m n}$

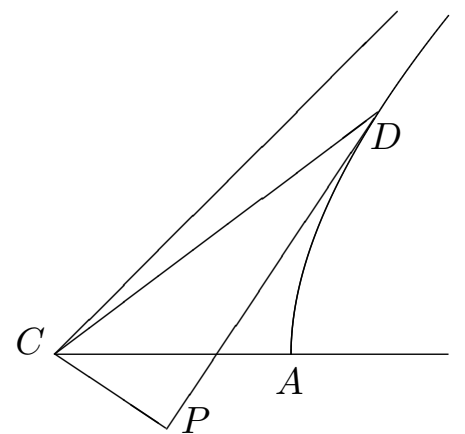

$D P-\operatorname{arc} A D=\int \sqrt{\frac{(m-n)^{2}-t^{2}}{(m+n)^{2}-t^{2}}} d t$

\section{première ellipse}

semi-axe transverse : $m+n$ semi-axe conjugué $: 2 \sqrt{m n}$

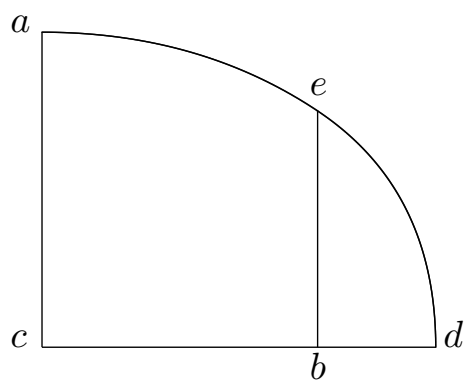

$\operatorname{arc} a e=\int \sqrt{\frac{(m+n)^{2}-t^{2}}{(m-n)^{2}-t^{2}}} d t$

\section{seconde ellipse}

semi-axe transverse : $m$

semi-axe conjugué : $n$

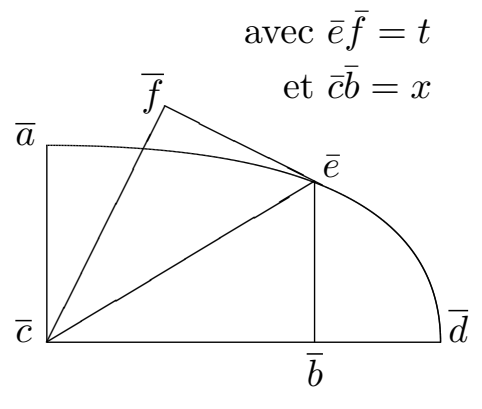

$t=g x \cdot \sqrt{\frac{m^{2}-x^{2}}{m^{2}-g x^{2}}}$ où $g=\frac{m^{2}-n^{2}}{m^{2}}$

FIG. 4 - Les trois lemmes de la démonstration de Landen

La phase préparatoire à la démonstration se déploie donc en trois moments.

(1) Landen considère d'abord une hyperbole de semi-axe transverse $m-n$ et de semi-axe conjugué $2 \sqrt{m n}$, et exprime la différence entre la tangente et l'arc sous la forme suivante

$$
D P-\operatorname{arc} A D=\int_{0}^{t} \sqrt{\frac{(m-n)^{2}-t^{2}}{(m+n)^{2}-t^{2}}} d t,
$$

\footnotetext{
${ }^{50}$ L'expression que donne Landen pour la différentielle irrationnelle concorde aussi bien, aux identifications près, avec la formule particulière de Maclaurin pour l'hyperbole équilatère (qui s'obtient en annulant le paramètre f) qu'avec l'expression générale de d'Alembert. Notons à cet égard que si Landen est réellement tributaire du résultat de caractère algébrique de d'Alembert, auquel il se réfère explicitement dès les premières lignes de son mémoire de 1771, c'est que la construction géométrique de la figure que proposait Maclaurin dans le cas de l'hyperbole équilatère ne pouvait pas être transposée telle quelle au cas d'une hyperbole de paramètres arbitraires, car la bissection de l'angle qui en est un maillon essentiel n'a plus lieu d'être dans le cas général. Il fallait donc emprunter un autre chemin et partir de la formule générale de d'Alembert pour parvenir à l'expression de l'excès de la tangente sur l'arc dans le cas d'une hyperbole de semi-axe transverse $m$ et de semi-axe conjugué $n$. Landen donne toutefois certaines indications qui permettent de rapporter la variable d'intégration $z$ à la figure géométrique et de préciser ainsi les bornes de l'intégrale, en fournissant de surcroît la clef du changement de variable qui devait conduire ensuite à la formule du mémoire de 1775. Il est en effet précisé que, si l'on pose la perpendiculaire $S P=p$, alors la variable d'intégration $z=\frac{p^{2}}{m}$, en sorte que $p$ et $z$ sont égaux à $m$ lorsque l'arc $A E$ et la tangente $E P$ s'annulent, et tendent vers 0 , lorsque l'arc et la tangente tendent vers l'infini. Par conséquent l'intégrale est prise entre les valeurs $m$ et $z$, où $0 \leq z \leq m$, ce qui s'accorde avec le fait que la différentielle soit négative.
} 
laquelle s'obtient à partir de l'expression donnée en 1771 au moyen d'un changement de variable $^{51}$.

(2) Puis, en appliquant la formule traditionnelle pour l'arc d'ellipse d'axes $a$ et $b$, à savoir $\int_{0}^{x} \frac{\sqrt{a^{2}-g x^{2}}}{\sqrt{a^{2}-x^{2}}}$ où $x$ est l'abscisse $c b$ (cf. Fig. 4) et $g=\frac{a^{2}-b^{2}}{a^{2}}$, au cas où les paramètres $a$ et $b$ sont respectivement égaux à $m+n$ et $2 \sqrt{m n}$, il détermine, à la faveur d'un changement de variable approprié $^{52}$, l'intégrale représentant la longueur d'arc mesurée à partir du petit axe d'une ellipse de semi-arc transverse $m+n$ et de semi-axe conjugué $2 \sqrt{m n}$,

$$
\operatorname{arc} a e=\int_{0}^{t} \sqrt{\frac{(m+n)^{2}-t^{2}}{(m-n)^{2}-t^{2}}} d t .
$$

(3) Enfin, dans le cas d'une ellipse de semi-axe transverse $m$ et de semi-axe conjugué $n$, Landen exprime, pour tout point de l'ellipse, la relation liant la tangente $t=\bar{e} \bar{f}$ à la courbe en ce point prolongée jusqu'à la perpendiculaire abaissée du centre de l'ellipse d'une part et l'abscisse $x=\bar{c} \bar{b}$ de ce même point d'autre part, soit

$$
t=g x \cdot \sqrt{\frac{m^{2}-x^{2}}{m^{2}-g x^{2}}} \quad \text { où } \quad g=\frac{m^{2}-n^{2}}{m^{2}} .
$$

Si l'on exprime la valeur du rayon $r=\overline{c e}$ à partir des coordonnées $\left(x, n \sqrt{1-\frac{x^{2}}{m^{2}}}\right)$ d'un point $\bar{e}$ de l'ellipse (cf. Fig. 4)

$$
r=\sqrt{n^{2}+\frac{\left(m^{2}-n^{2}\right)}{m^{2}} x^{2}}
$$

la relation algébrique entre la tangente et l'abscisse s'obtient par le calcul à partir de la proposition de Maclaurin exprimant que "la fluxion de l'arc est à la fluxion du rayon comme le rayon est à la tangente", en substituant dans l'équation $t=\frac{r d r}{d s}$ les expressions en $x$ correspondant au rayon et aux deux différentielles ${ }^{53}$.

Sur la base de ces trois lemmes préparatoires, la démonstration proprement dite procède alors selon le schéma suivant. De l'équation algébrique liant la tangente et l'abscisse (cf. Eq. 13),

\footnotetext{
${ }^{51}$ En assignant, dans la formule de 1771 (vide supra), les valeurs $m-n$ et $2 \sqrt{m n}$ aux semi-axes transverse et conjugué, respectivement, on obtient l'expression

$$
D P-\operatorname{arc} A D=\int_{m-n}^{z} \frac{-\frac{1}{2} \sqrt{m-n} \sqrt{z} d z}{\sqrt{4 m n+\left(\frac{(m-n)^{2}-4 m n}{m-n}\right) z-z^{2}}},
$$

qu'on peut transformer en tirant parti de l'indication, donnée par Landen, que la perpendiculaire $C P$ à la tangente est telle que $C P=\sqrt{(m-n)^{2}-t^{2}}$. En rapportant en effet cette égalité aux stipulations de 1771, à savoir que $C P=p$ et $z=\frac{p^{2}}{m-n}$, on déduit le changement de variable $z=\frac{(m-n)^{2}-t^{2}}{m-n}$, qui permet de transformer l'intégrale ci-dessus en cette autre $\int_{0}^{t} \sqrt{\frac{(m-n)^{2}-t^{2}}{(m+n)^{2}-t^{2}}} d t$, prise entre les bornes 0 et $t$.

${ }^{52}$ En substituant les valeurs $m+n$ et $2 \sqrt{m n}$, respectivement, aux paramètres $a$ et $b$ pour les semi-axes transverse et conjugué, on obtient l'intégrale

$$
\operatorname{arc} a e=\int_{0}^{x} \frac{\sqrt{(m+n)^{2}-\frac{(m-n)^{2}}{(m+n)^{2}} x^{2}}}{\sqrt{(m+n)^{2}-x^{2}}} d x
$$

que le changement de variable $x=\frac{m+n}{m-n} t$ transforme en cette autre $\int_{0}^{t} \sqrt{\frac{(m+n)^{2}-t^{2}}{(m-n)^{2}-t^{2}}} d t$ prise entre les bornes 0 et $t$. ${ }^{53}$ Le calcul donne immédiatement $d r=\frac{\left(\frac{m^{2}-n^{2}}{m^{2}}\right) x d x}{\sqrt{n^{2}+\frac{\left(m^{2}-n^{2}\right)}{m^{2}} x^{2}}}$, ainsi que $d s=\sqrt{(d x)^{2}+(d y)^{2}}=\sqrt{1+\frac{n^{2} x^{2}}{m^{2}\left(m^{2}-x^{2}\right)}} d x$, dont se déduit aussitôt l'équation recherchée.
} 
Landen tire une équation biquadratique en $x$

$$
\left(x^{2}-\left(\frac{m^{2} g+t^{2}}{2 g}\right)\right)^{2}=\frac{\left(m^{2}-n^{2}\right)^{2}-2\left(m^{2}+n^{2}\right) t^{2}+t^{4}}{(2 g)^{2}}
$$

qu'il résout de façon à obtenir une expression de $x^{2}$ en fonction de $t$

$$
x^{2}=\frac{m^{2} g+t^{2}}{2 g}-\frac{\sqrt{\left(m^{2}-n^{2}\right)^{2}-2\left(m^{2}+n^{2}\right) t^{2}+t^{4}}}{2 g},
$$

laquelle fournit, par différentiation, une expression de $2 x d x$ en fonction de $t$ et de $d t$

$$
x d x=\frac{t d t}{2 g}+\frac{\left(m^{2}+n^{2}\right) t d t-t^{3} d t}{2 g \sqrt{\left(m^{2}-n^{2}\right)^{2}-2\left(m^{2}+n^{2}\right) t^{2}+t^{4}}} .
$$

Mais en posant l'arc $z=\bar{a} \bar{e}$ (cf. Fig. 4)) et en combinant l'expression usuelle pour l'élément d'arc $d z$ d'une ellipse de semi-axes $m, n$ avec l'expression pour la tangente établie ci-dessus (cf. lemme (3)),

$$
d z=\sqrt{\frac{m^{2}-g x}{m^{2}-x^{2}}} d x \quad \text { et } \quad t=g x \cdot \sqrt{\frac{m^{2}-x^{2}}{m^{2}-g x^{2}}},
$$

Landen obtient la relation $t d z=g x d x$, dont il tire une nouvelle expression pour l'élément d'arc

$$
d z=\frac{d t}{2}+\frac{\left(m^{2}+n^{2}\right) d t-t^{2} d t}{2 \sqrt{\left(m^{2}-n^{2}\right)^{2}-2\left(m^{2}+n^{2}\right) t^{2}+t^{4}}} .
$$

Une identité remarquable permet alors de factoriser le radical au dénominateur

$$
\left(m^{2}-n^{2}\right)^{2}-2\left(m^{2}+n^{2}\right) t^{2}+t^{4}=\left[(m-n)^{2}-t^{2}\right] \cdot\left[(m+n)^{2}-t^{2}\right],
$$

ce qui constitue la clef du théorème de rectification, puisqu'en divisant en deux parties égales le terme fractionnaire avec radical,

$$
d z=\frac{d t}{2}+\frac{\left[\left(m^{2}+n^{2}\right)-t^{2}\right] d t}{4 \sqrt{\left[(m-n)^{2}-t^{2}\right] \cdot\left[(m+n)^{2}-t^{2}\right]}}+\frac{\left[\left(m^{2}+n^{2}\right)-t^{2}\right] d t}{4 \sqrt{\left[(m-n)^{2}-t^{2}\right] \cdot\left[(m+n)^{2}-t^{2}\right]}}
$$

on fait apparaître les différentielles ${ }^{54}$

$$
d z=\frac{d t}{2}+\frac{1}{4} \sqrt{\frac{(m+n)^{2}-t^{2}}{(m-n)^{2}-t^{2}}} \cdot d t+\frac{1}{4} \sqrt{\frac{(m-n)^{2}-t^{2}}{(m+n)^{2}-t^{2}}} \cdot d t,
$$

lesquelles correspondent respectivement à l'excès de la tangente sur l'arc d'hyperbole (lemme (1)) et à l'arc d'ellipse de semi-axes $m+n, 2 \sqrt{m n}$ (lemme (2)). Par intégration, on obtient alors la relation géométrique entre les arcs suivants

$$
\operatorname{arc} \bar{a} \bar{e}=\frac{\bar{e} \bar{f}}{2}+\frac{D P-\operatorname{arc} A D}{4}+\frac{\operatorname{arc} a e}{4},
$$

et par suite l'expression pour l'arc hyperbolique

$$
\operatorname{arc} A D=\operatorname{arc} a e-4 \operatorname{arc} \bar{a} \bar{e}+D P+2 \bar{e} \bar{f},
$$

qui fournit le théorème de rectification que Landen découvre comme par surcroît au cours des recherches qu'il consacre à l'excès de la tangente sur l'arc d'hyperbole : "Thus, beyond my expectation, I find that the hyperbola may in general be rectified by means of two ellipses" 55 .

\footnotetext{
${ }^{54}$ Il suffit pour cela d'utiliser les identités remarquables $(m-n)^{2}=m^{2}+n^{2}-2 m n$ et $(m+n)^{2}=m^{2}+n^{2}+2 m n$. ${ }^{55}[45$, p. 285].
} 


\subsection{Du statut implicite de la transformation de Landen à son explicitation rétrospective}

Le théorème de rectification a souvent été interprété, tant par les mathématiciens que par les historiens des mathématiques, à la lumière de la théorie des intégrales elliptiques développée par la suite, comme l'origine de la transformation dite de Landen. Cette interprétation se fonde sur le remarque selon laquelle les deux ellipses auxquelles le résultat de Landen ramène en définitive ${ }^{56}$ l'arc d'hyperbole sont liées par une relation particulière qui peut être caractérisée par le fait que les semi-axes de l'une des deux ellipses sont respectivement les moyennes arithmétique $\left(\frac{m+n}{2}\right)$ et géométrique $(\sqrt{m n})$ des semi-axes $m, n$ de l'autre. L'importance des propriétés relatives à la moyenne arithmético-géométrique pour la théorie des intégrales elliptiques ne devait être reconnue que plus tard, après que les travaux de Gauss se rapportant à cet ordre de questions eurent été publiés ${ }^{57}$. La question se pose donc de savoir en quel sens Landen a pu lui-même appréhender comme une forme stable, et constituer comme objet mathématique indépendant, la transformation enveloppée dans la relation entre les deux ellipses de son théorème de rectification.

Dans la reconstruction qu'il propose de la démarche de Landen ([63]), et dont il reconnaît d'ailleurs lui-même qu'elle est largement tributaire du point de vue de Legendre, G.-N Watson (1886-1965) par exemple ignore délibérément ce problème historiographique pour privilégier, fût-ce au prix de distorsions historiques importantes, la netteté de l'image ainsi restituée des origines du sujet qui l'occupait au début des années mil neuf cent trente, à savoir la multiplication complexe et la recherche des modules singuliers.

Pairs of ellipses whose semi-axes are related in the manner of the two ellipses of this problem are to be connected by Landen's transformation. In the hands of Legendre, the transformation became a most powerful method for computing elliptic integrals; and the transformation made possible the theory of more general transformations, leading up to the theories of modular equations, complex multiplication, and singular moduli. [63, p. 14-5]

La présentation de Watson se signale toutefois par l'absence significative d'un maillon important dans la reconstitution ainsi suggérée. Rien n'est dit en effet pour expliquer la transition des arcs d'ellipse correspondant à des intégrales de seconde espèce dans la classification de Legendre à la forme traditionnellement reçue pour la transformation de Landen qui porte sur des intégrales de première espèce. Au siècle précédent, dans une note consacrée à Landen de L'Encyclopedia Britannica (1882), Cayley avait toutefois fait entendre une note sensiblement différente en se gardant d'imputer l'identification de la transformation au mathématicien britannique.

Landen's capital discovery is that of the theorem known by his name (obtained in its complete form in the memoir of 1775, and reproduced in the first volume of the Mathematical Memoirs) for the expression of the arc of an hyperbola in terms of two elliptic arcs. To find this, he integrates a differential equation derived from the equation

$$
t=g x \sqrt{\frac{m^{2}-x^{2}}{m^{2}-g x^{2}}},
$$

\footnotetext{
${ }^{56}$ Selon la lettre du texte de Landen, il est en fait question d'une ellipse de semi-axes $m+n$ et $2 \sqrt{m n}$, mais la lecture rétrospective lui substitue une ellipse de semi-axes $\frac{m+n}{2}, \sqrt{m n}$, pour pouvoir mettre en relief la forme d'une transformation distinguée entre les intégrales elliptiques correspondantes. Cette inflexion est rendue possible par le fait que les deux ellipses s'obtiennent l'une à partir de l'autre par homothétie.

${ }^{57}$ Sur la moyenne arithmético-géométrique de Gauss, cf. [28], et sur les liens entre la transformation de Gauss et la transformation de Landen, cf. [10, p. 279] et [1, p. 590]. Gauss étudie les propriétés de l'intégrale $I(a, b)=$ $\int_{0}^{\frac{\pi}{2}} \frac{d \phi}{\sqrt{a^{2} \cos ^{2} \phi+b^{2} \sin ^{2} \phi}}$ et montre que $I(a, b)=I\left(\frac{a+b}{2}, \sqrt{a b}\right)$, d'où s'obtient par itération la valeur de la moyenne arithmético-géométrique $\mu(a, b)=\frac{\pi / 2}{I(a, b)}$. Pour appliquer le théorème de Gauss aux intégrales elliptiques complètes de première espèce, il suffit de poser $k=\frac{a-b}{a+b}$, car alors $I(a, b)=\frac{1}{a} \cdot F\left(\frac{2 \sqrt{k}}{1+k}, \frac{\pi}{2}\right)$, de sorte que le théorème de Gauss peut être reformulé de la manière suivante $: F\left(\frac{2 \sqrt{k}}{1+k}, \frac{\pi}{2}\right)=(1+k) F\left(k, \frac{\pi}{2}\right)$. La transformation de Gauss s'obtient donc comme un cas particulier de la transformation de Landen appliquée à des intégrales complètes.
} 
interpreting geometrically in an ingenious and elegant manner three integrals which present themselves. If in the foregoing equation we write $m=1, g=k^{2}$, and instead of $t$ consider the new variable $y=t \div\left(1-k^{\prime}\right)$, then

$$
y=\left(1+k^{\prime}\right) x \sqrt{\frac{1-x^{2}}{1-k^{2} x^{2}}},
$$

which is the form known as Landen's transformation in the theory of elliptic functions; but his investigation does not lead him to obtain the equivalent of the resulting differential equation

$$
\frac{d y}{\sqrt{1-y^{2} .1-\lambda^{2} y^{2}}}=\frac{\left(1+k^{\prime}\right) d x}{\sqrt{1-x^{2} \cdot 1-k^{2} x^{2}}}, \quad \text { where } \quad \lambda=\frac{1-k^{\prime}}{1+k^{\prime}}
$$

due[,] it would appear[,] to Legendre and which (over and above Landen's own beautiful result) gives importance to the theorem as leading directly to the quadric transformation of an elliptic integral in regard to the modulus. ${ }^{58}$

Du côté des historiens des mathématiques, la même prudence méthodologique prévaut. Roger Cooke ([9]) signale ainsi ce problème des conditions d'identification.

In discovering this relationship [i.e. la relation entre les deux ellipses du théorème de rectification], Landen implicitly gave the first example of the technique known as transformation of elliptic integrals, which was to form an essential part of both the theoretical and the computational aspect of elliptic functions. ${ }^{59}$

Reconnaître que la transformation de Landen telle qu'elle fut dégagée par la suite était implicite dans le texte de Landen nous conduit naturellement à nous interroger sur les conditions de son explicitation. Sur la base d'une analyse de l'ensemble de l'œuvre mathématique de Landen, Gwynedd Green et Winter ([30]) privilégiaient une position plus tranchée en soutenant qu'il serait sans doute illusoire d'imputer à Landen une claire compréhension de la relation entre les deux ellipses comme transformation entre intégrales elliptiques.

While his interest and application in these directions were acute, Landen failed to realize that the whole of his analytical transformations were particular cases of one general one, now known as the Landen transformations. ${ }^{60}$

Dans un sens analogue, Niccolò Guicciardini considère qu'il n'y a pas à proprement parler chez Landen de théorie des intégrales elliptiques pour autant que l'intérêt chez lui se portait davantage sur la relation géométrique entre l'arc d'hyperbole et l'arc d'ellipse que sur une quelconque transformation entre intégrales elliptiques ${ }^{61}$.

\section{Les premiers mémoires de Legendre sur l'intégration par arcs d'ellipse}

Dans le premier de ses deux mémoires sur les méthodes d'intégration par arcs d'ellipse publiés pour l'année 1786 par l'Académie royale des sciences de Paris, Legendre démontre la rectification de l'hyperbole au moyen de deux ellipses, indépendamment de Landen et selon une approche différente fondée sur la variation continue des intégrales par rapport au module. En outre, à la

\footnotetext{
${ }^{58}[8$, p. 584$]$.

$59[9$, p. 533$]$, nous soulignons l'adverbe.

$60[30$, p. 9$]$.

${ }^{61}$ Cf. [29, p. 89] : "[Landen's] problem was not that of integrating functions of the form $\left(\left(1-x^{2}\right)\left(1-q^{2} x^{2}\right)\right)^{-1 / 2}$, and he never expressed Landen's transformations in the form known today.".
} 
différence de son prédécesseur, il interprète et utilise ce résultat dans la perspective d'un calcul approché des transcendantes qui le conduit à élaborer des tables numériques associant aux arcs d'ellipse les coefficients de leur différence partielle relativement au module.

Nous démontrerons facilement que les arcs d'hyperbole dépendent entièrement des arcs d'ellipse, \& n'offrent point une espèce particulière de transcendante. Il y a donc une multitude d'intégrales qu'on rapportait à la rectification de l'ellipse \& de l'hyperbole, \& qui ne dépendent que de celle de l'ellipse. Cette observation m'a fait penser qu'il y aurait de l'avantage à introduire dans le calcul les arcs d'ellipse, à peu près comme les arcs de cercle \& les logarithmes. D'après les formes que j'ai choisies, je pense que les géomètres trouveront la chose praticable \& même commode dans bien des cas. Mais il ne suffit pas d'indiquer un résultat, il faut être en état de l'évaluer avec toute l'approximation nécessaire. Il serait donc très avantageux que d'habiles calculateurs prissent la peine de dresser des tables d'arcs d'ellipse avec l'étendue convenable, \& qu'ils joignissent en même temps à chaque arc la valeur du coefficient aux différences partielles, dont on verra que l'usage est très fréquent. ${ }^{62}$

Dans le second mémoire, Legendre s'attache alors à démontrer à partir de ses principes propres le théorème de Landen dont il dit n'avoir pris connaissance que dans l'intervalle séparant les deux communications, ce qui le conduit à produire une interprétation du théorème de rectification qui met en relief la transformation de Landen encore implicite dans le théorème du mathématicien anglais.

\subsection{Le calcul des arcs d'ellipse}

Dans le premier mémoire, Legendre considère des ellipses de semi-axes 1 et $b$, avec $0<b<1$ (cf. Fig. 5) et introduit une notation spécifique pour les formules usuelles correspondant aux arcs mesurés respectivement à partir des extrémités du semi-axe conjugué et du semi-axe transverse.

$$
\begin{aligned}
& \operatorname{arc} B M=\int_{0}^{\phi} \sqrt{1-c^{2} \sin ^{2} \phi} \cdot d \phi=E(c, \phi), \\
& \operatorname{arc} A M=\int_{0}^{\phi} \sqrt{1-c^{2} \cos ^{2} \phi} \cdot d \phi=F(c, \phi),
\end{aligned}
$$

où $c=\sqrt{1-b^{2}}$ et où l'angle $\phi$ que Legendre nomme l'amplitude de l'arc $E$ (respectivement $F^{63}$ ) pour le module $c$ correspond à l'arc $D Z$ (respectivement $A Z$ ) sur le cercle unité. Quoique ces deux fonctions se rapportent de manière évidente l'une à l'autre, en sorte qu'on pourrait n'en introduire qu'une seule, Legendre les admet toutes les deux pour des raisons qui tiennent à la commodité du calcul.

Pour pouvoir établir des tables d'arcs, Legendre cherche alors à calculer avec toute l'approximation nécessaire la valeur de $E(c, \phi)$ et de $F(c, \phi)$ pour les valeurs de $c$ et de $\phi$ pour lesquelles les formules obtenues par développement en série infinie sont le plus convergentes, et à y ramener celles qui correspondent à des valeurs de $c$ et de $\phi$ pour lesquelles ces mêmes formules cessent d'être convergentes, en utilisant certaines relations appropriées entre les arcs de différentes ellipses.

Plus généralement, Legendre se propose de réduire progressivement aux arcs d'ellipse des classes de plus en plus étendues de différentielles, en commençant par les différentielles de la forme

$$
d \phi \sqrt{f+g \sin ^{2} \phi} \quad \text { et } \quad d \phi \sqrt{f+g \cos ^{2} \phi}
$$

\footnotetext{
${ }^{62}[46$, p. 616$]$.

${ }^{63}$ Notons incidemment que les notations de 1786 doivent être distinguées de celles que Legendre adopte par la suite dans le mémoire de 1793 et les traités ultérieurs de 1811 et de 1825 , où la lettre $E$ est maintenue pour les arcs d'ellipse et plus généralement pour les intégrales elliptiques de seconde espèce, mais où la lettre $F$ en revanche est utilisée pour désigner le type des intégrales elliptiques de première espèce, et non plus comme ici les arcs d'ellipse mesurés à partir de l'extrémité du semi-axe transverse.
} 


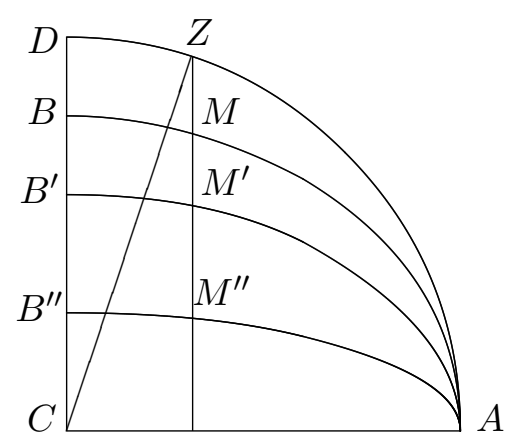

FIG. 5 - La variation continue dans la famille d'ellipses

où $f$ et $g$ sont de valeur et de signe arbitraires. Si la réduction est évidente dans le cas de paramètres strictement positifs où elle se ramène immédiatement aux formules ${ }^{64}$

$$
\begin{aligned}
& \int d \phi \sqrt{1+\frac{c^{2}}{1-c^{2}} \cos ^{2} \phi}=\frac{1}{\sqrt{1-c^{2}}} E(c, \phi) \\
& \int d \phi \sqrt{1+\frac{c^{2}}{1-c^{2}} \sin ^{2} \phi}=\frac{1}{\sqrt{1-c^{2}}} F(c, \phi),
\end{aligned}
$$

le traitement du cas général l'amène en revanche à introduire, outre les arcs d'ellipse, les dérivées partielles des intégrales qui les représentent. Il considère en effet les deux cas suivants

$$
d \phi \sqrt{g \cos ^{2} \phi-f} \quad \text { et } \quad d \phi \sqrt{f-g \sin ^{2} \phi}
$$

et montre qu'une substitution permet de les ramener aux arcs d'ellipse. Si $f$ et $g$ sont supposés strictement positifs et si $g>f$, alors le rapport des deux paramètres $f / g$ étant compris entre 0 et 1 , peut être représenté par le carré du cosinus d'un nouvel angle $\alpha$

$$
d \phi \sqrt{g \cos ^{2} \phi-f}=\sqrt{g} d \phi \sqrt{\cos ^{2} \phi-\cos ^{2} \alpha} .
$$

Legendre propose alors la substitution suivante, l'angle $\alpha$ ayant valeur de paramètre,

$$
\sin \phi=\sin \alpha \cdot \sin \psi
$$

de sorte qu'en posant $c=\sin \alpha$, on obtienne

$$
d \phi \sqrt{\cos ^{2} \phi-\cos ^{2} \alpha}=\frac{c^{2} \cdot \cos ^{2} \psi \cdot d \psi}{\sqrt{1-c^{2} \sin ^{2} \psi}} .
$$

Mais il est maintenant possible de reconnaître dans l'intégrale correspondante une combinaison de la fonction $E(c, \psi)$ et de sa dérivée partielle $\frac{d E}{d c}$

$$
\int \frac{c^{2} \cdot \cos ^{2} \psi \cdot d \psi}{\sqrt{1-c^{2} \sin ^{2} \psi}}=c^{2} E(c, \psi)+\left(1-c^{2}\right) c \cdot \frac{d E}{d c}
$$

ce qui achève la réduction souhaitée de la différentielle de la forme $d \phi \sqrt{g \cos ^{2} \phi-f}$.

\footnotetext{
${ }^{64} \mathrm{Si} f$ et $g$ sont strictement positifs, une simple factorisation suffit à faire apparaître le module de l'arc d'ellipse auquel on se ramène, car $d \phi \sqrt{f+g \sin ^{2} \phi}=\sqrt{f} \cdot d \phi \sqrt{1+\frac{g}{f} \sin ^{2} \phi}$ et $\frac{g}{f}=\frac{c^{2}}{1-c^{2}}$.
} 
Dans le même ordre d'idées, Legendre élargit encore les méthodes d'intégration par arcs d'ellipses à d'autres types de différentielles, par exemple en montrant plus généralement que les intégrales

$$
\int \Delta^{2 m+1} d \phi, \quad \int \frac{d \phi}{\Delta^{2 m+1}}
$$

où $\Delta=\sqrt{1-c^{2} \sin ^{2} \phi}$, admettent des expressions normalisées dont on peut éliminer toutes les dérivées partielles de $E$ au-delà du premier ordre et sont donc réductibles à la forme $\alpha E+\beta \frac{d E}{d c}+\xi$, où $\alpha$ et $\beta$ ne dépendent que du module $c$ et $\xi$ est une fonction algébrique du module et de $\sin \phi$.

\subsection{La rectification de l'hyperbole selon Legendre}

À titre d'exemple privilégié de l'application de ses méthodes d'intégration par arcs d'ellipse, Legendre donne une première rectification de l'hyperbole qui se démarque nettement de celle de Landen et dont nous nous proposons d'expliciter les étapes successives en restituant les constructions géométriques sous-jacentes que, dans sa présentation analytique, Legendre fait passer au second plan.

Soit l'excentricité $C A=1$, le demi-axe transverse $C F=c$, le demi-axe conjugué $C P=$ $b=\sqrt{1-c^{2}}$, l'abscisse $C P^{\prime}=\frac{c}{\cos \phi}$, l'ordonnée $P^{\prime} M=b \tan \phi$; on aura l'arc

$$
F M^{\prime}=\int \frac{d \phi}{\cos ^{2} \phi} \sqrt{1-c^{2} \cos ^{2} \phi}
$$

quantité qui s'intègre par les méthodes précédentes. ${ }^{65}$

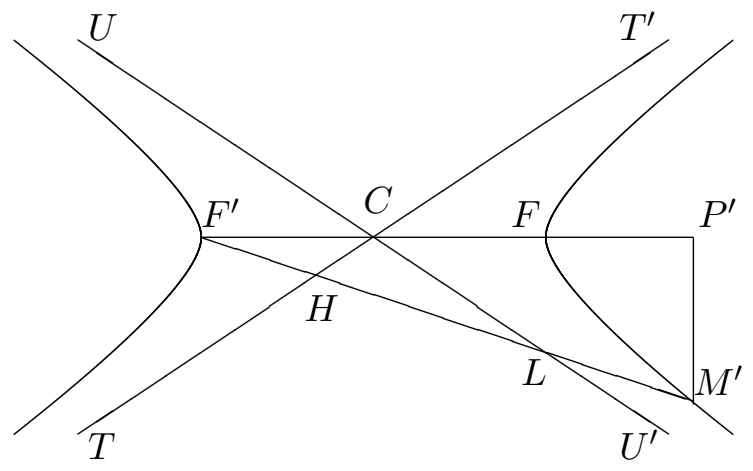

FiG. 6 - La construction cissoïdale de l'hyperbole

Legendre se réfère ici implicitement à la construction cissoïdale de l'hyperbole (cf. Fig. 6). L'hyperbole équilatère de sommets $F, F^{\prime}$ peut en effet être envisagée comme le lieu géométrique des points $M^{\prime}$ tels que l'on ait

$$
F^{\prime} H=L M^{\prime}
$$

où $H$ et $L$ sont les points où le rayon mobile issu du sommet $F^{\prime}$ rencontre les asymptotes $T T^{\prime}$, $U U^{\prime}$ respectivement. Les coordonnées du point $M^{\prime}(\xi, \eta)$ s'expriment ${ }^{66}$ alors en fonction de l'angle $\omega=\angle F F^{\prime} M^{\prime}$ :

$$
\xi=\frac{c\left(b^{2}+c^{2} \tan ^{2} \omega\right)}{b^{2}-c^{2} \tan ^{2} \omega}, \quad \eta=\frac{b(2 b c \tan \omega)}{b^{2}-c^{2} \tan ^{2} \omega},
$$

\footnotetext{
${ }^{65}[46$, p. 634$]$.

${ }^{66}$ On détermine en premier lieu les coordonnées des points $H$ et $L$ en fonction de l'angle $\omega$ en prenant l'intersection de la droite mobile $F^{\prime} M^{\prime}$ issue du sommet $F^{\prime}(-c, 0)$ et de pente $\tan \omega$ avec les asymptotes $T T^{\prime}$ et $U U^{\prime}$ d'équations $x= \pm \frac{b}{c} y$. On obtient ainsi $H\left(\frac{c^{2} \tan \omega}{b-c \tan \omega}, \frac{b c \tan \omega}{b-c \tan \omega}\right)$ et $L\left(\frac{-c^{2} \tan \omega}{b+c \tan \omega}, \frac{b c \tan \omega}{b+c \tan \omega}\right)$ et la condition $F^{\prime} H=L M^{\prime}$ permet de trouver l'expression des coordonnées $(\xi, \eta)$ de $M^{\prime}$.
} 
de sorte que par un changement de variable approprié

$$
\tan \frac{\phi}{2}=\frac{c}{b} \tan \omega
$$

on obtienne

$$
\xi=\frac{c\left(1+\tan ^{2} \frac{\phi}{2}\right)}{1-\tan ^{2} \frac{\phi}{2}}=\frac{c}{\cos \phi}, \quad \eta=\frac{b\left(2 \tan \frac{\phi}{2}\right)}{1-\tan ^{2} \frac{\phi}{2}}=b \tan \phi,
$$

qui correspondent aux coordonnées indiquées par Legendre pour le point $M^{\prime}: C P^{\prime}=\frac{c}{\cos \phi}$ et $P^{\prime} M=b \tan \phi$, dont on tire ensuite l'élément d'arc $\frac{d \phi}{\cos ^{2} \phi} \sqrt{1-c^{2} \cos ^{2} \phi}$.

Legendre construit alors une ellipse sur laquelle il détermine un arc $A M$ répondant à la même amplitude $\phi$ que l'arc hyperbolique $F M^{\prime}$.

Décrivons une ellipse $A M B$ qui soit telle, par rapport à l'hyperbole $F M^{\prime}$, que le sommet de l'une soit le foyer de l'autre; prenons de plus $C X=C P^{\prime}$, afin de déterminer sur l'ellipse l'arc $A M$, qui réponde à la même valeur de $\phi$ que l'arc hyperbolique $F M^{\prime}$; on aura

$$
A M \text { ou } F=\int d \phi \sqrt{1-c^{2} \cos ^{2} \phi}
$$

\& de là

$$
F M^{\prime}=\tan \phi \sqrt{1-c^{2} \cos ^{2} \phi}-c^{2} F-b^{2} c \frac{d F}{d c}
$$

quantité où la partie algébrique

$$
\tan \phi \sqrt{1-c^{2} \cos ^{2} \phi}
$$

représente la tangente en $M^{\prime}$ ou en $M$ terminée au premier axe. Il est donc évident que la rectification de l'hyperbole dépend de celle de l'ellipse, \& n'offre point de transcendante particulière. ${ }^{67}$

Legendre propose ainsi une construction à la règle et au compas du point $M$ sur l'ellipse qu'il faut reconstituer à partir de la figure et de la seule indication textuelle qu'il donne : "prenons de plus $C X=C P$ "'.

Soient donc une hyperbole de semi-axes $C F=c, C B=b=\sqrt{1-c^{2}}$ et une ellipse de semiaxes $C A=1, C B$, par conséquent telles que le sommet de l'une soit le foyer de l'autre. Prenons sur l'hyperbole un point $M^{\prime}$ d'abscisse $C P^{\prime}$ et traçons le cercle de centre $C$ et de rayon $C P^{\prime}$ qui rencontre la verticale élevée au dessus du sommet $F$ au point $X$. La droite $C X$ coupe le cercle de centre $C$ et de rayon $C A=1$ en $Z$ d'abscisse $C P$. La droite $P Z$ coupe l'ellipse en un point $M$ qui détermine un $\operatorname{arc} A M$ qui répond à la même amplitude $\phi$ que l'arc hyperbolique $F M^{\prime}$.

$\mathrm{Si}$, comme précédemment, nous posons $\angle A C Z=\phi$ et $\angle F F^{\prime} M^{\prime}=\omega$, la correction de la construction géométrique de Legendre se déduit immédiatement de la relation liant les angles $\omega$ et $\phi$, à savoir

$$
\tan \frac{\phi}{2}=\frac{c}{b} \tan \omega
$$

de sorte qu'elle fournit en retour ${ }^{68}$ l'interprétation géométrique sous-jacente au changement de variable permettant d'obtenir l'expression de l'arc hyperbolique $F M^{\prime}$ répondant à la même

\footnotetext{
${ }^{67}$ [46, p. 634-5]. Prenons garde ici à l'ambiguïté des notations adoptées par Legendre, lequel n'hésite pas à désigner au moyen de la même lettre $F$ un point de la figure, à la fois foyer de l'ellipse et sommet de l'hyperbole, et l'arc d'ellipse mesure à partir de l'extrémité de l'axe transverse.

${ }^{68}$ Le triangle $C F X$ est rectangle en $F$ et de côtés $\left(c, \sqrt{x^{2}-c^{2}}, x\right)$, par suite tan $\phi=\frac{\sqrt{x^{2}-c^{2}}}{c}$. Le triangle $F P^{\prime} M^{\prime}$, rectangle en $P^{\prime}$, a pour côtés $F P^{\prime}=x+c$ et $P^{\prime} M^{\prime}=y$, par conséquent $\tan \omega=\frac{y}{x+c}$. En utilisant le fait que les coordonnées $x, y$ du point $M^{\prime}$ satisfont à l'équation de l'hyperbole $\frac{x^{2}}{c^{2}}-\frac{y^{2}}{b^{2}}=1$, il est alors facile de montrer que $\tan \frac{\phi}{2}=\frac{c}{b} \tan \omega$.
} 


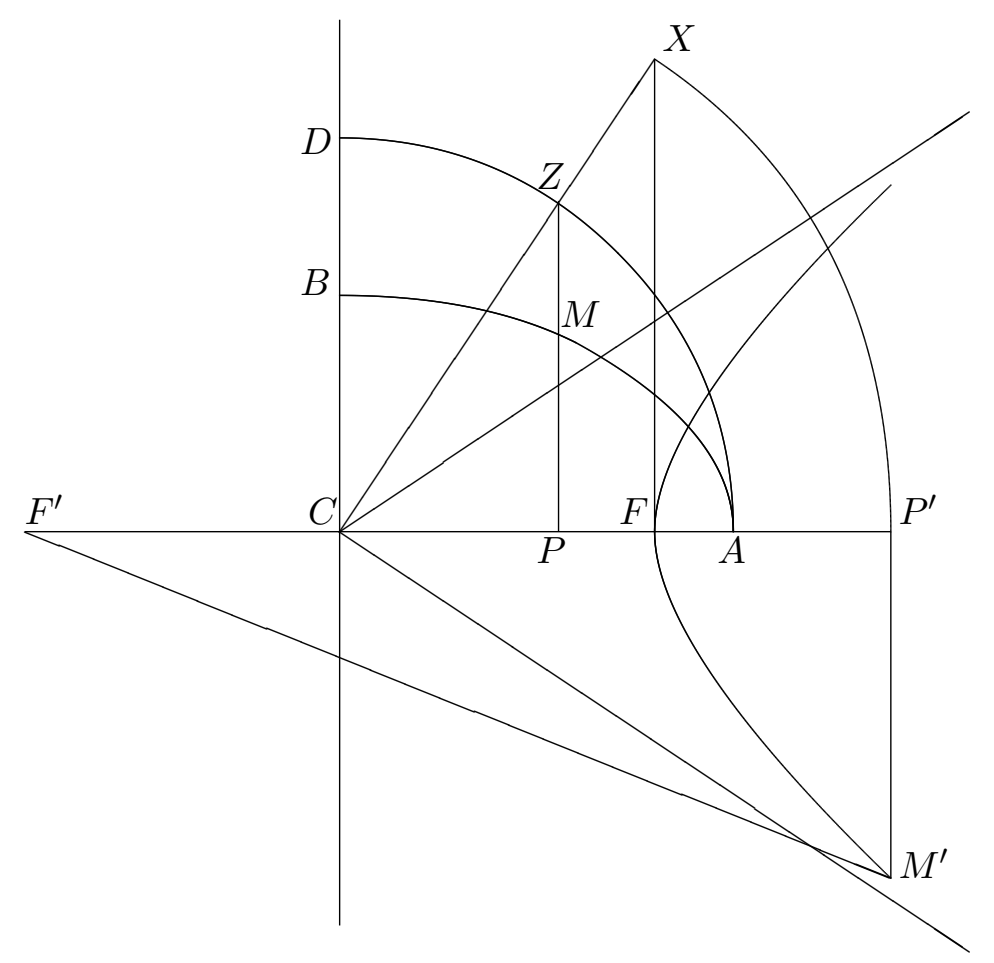

FIG. 7 - La construction de Legendre

amplitude $\phi$ que l'arc d'ellipse $A M$, car

$$
\operatorname{arc} F M^{\prime}=\int_{0}^{\phi} \frac{d \phi}{\cos ^{2} \phi} \sqrt{1-c^{2} \cos ^{2} \phi} \quad \text { et } \quad \operatorname{arc} A M=\int_{0}^{\phi} d \phi \sqrt{1-c^{2} \cos ^{2} \phi}
$$

La rectification de l'hyperbole s'obtient alors en intégrant par parties l'intégrale exprimant l'arc $F M^{\prime}$,

$$
\int \frac{d \phi}{\cos ^{2} \phi} \sqrt{1-c^{2} \cos ^{2} \phi}=\tan \phi \cdot \sqrt{1-c^{2} \cos ^{2} \phi}-\int \frac{c^{2} \sin ^{2} \phi}{\sqrt{1-c^{2} \cos ^{2} \phi}} d \phi
$$

puis en opérant une série de substitutions ${ }^{69}$ qui permettent d'exprimer les intégrales ci-dessus comme combinaisons des quantités ${ }^{70} F$ et $\frac{d F}{d c}$,

$$
\operatorname{arc} F M^{\prime}=\tan \phi \sqrt{1-c^{2} \cos ^{2} \phi}-c^{2} F-b^{2} c \frac{d F}{d c}
$$

ou, selon une expression équivalente que Legendre prend soin de donner aussi "pour plus d'uniformité",

$$
\operatorname{arc} F M^{\prime}=\tan \phi \sqrt{1-c^{2} \sin ^{2} \phi}-c^{2} E-b^{2} c \frac{d E}{d c},
$$

si l'on exprime l'arc d'hyperbole au moyen de l'arc d'ellipse compté depuis le petit axe.

\footnotetext{
${ }^{69}$ En posant $\Gamma=\sqrt{1-c^{2} \cos ^{2} \phi}$, on remarque en effet que $\frac{d \Gamma}{d c}=\frac{1}{c}\left(\Gamma-\frac{1}{\Gamma}\right)$, d'où Legendre tire la relation $\int \frac{d \phi}{\sqrt{1-c^{2} \cos ^{2} \phi}}=F-c \frac{d F}{d c}$ qui permet de ramener les intégrales aux arcs d'ellipse.

${ }^{70}$ Comme nous l'avons signalé précédemment, il faut prendre soin de ne pas confondre ici le point $F$ et l'arc d'ellipse $F$.
} 


\subsection{La relecture par Legendre du théorème de Landen}

Dans le second mémoire, Legendre revient sur le théorème de rectification obtenu précédemment et en souligne l'utilité qu'il lie étroitement à la constitution de tables numériques supposées fournir, pour différents degrés d'amplitude et d'excentricité, les arcs d'ellipse et les coefficients aux différences partielles associés. Mais il mentionne désormais le théorème de Landen qu'il compare à son propre résultat du point de vue de l'efficacité des méthodes de calcul correspondantes.

Depuis la lecture de ce mémoire ([46]), j'ai appris que M. Landen, membre de la Société royale de Londres, s'était occupé du même objet avec beaucoup de succès, (...)

Le résultat de M. Landen est que tout arc d'hyperbole se rectifie immédiatement par le moyen de deux arcs d'ellipse : proposition extrêmement intéressante, \& d'autant plus remarquable qu'elle est le fruit d'une transformation très adroite, qui avait échappé à tous ceux qui se sont occupés de ces objets.

Il n'est pas douteux que la découverte de M. Landen ne puisse procurer beaucoup d'élégance aux intégrations qui dépendent des arcs d'hyperbole; mais il me semble que les Tables, telles que je les ai proposées, où l'on trouverait le coefficient aux différences partielles à côté de chaque arc, seraient plus commodes dans les applications que l'emploi de deux arcs d'ellipses, dont les amplitudes et les excentricités seraient différentes, \& qui entraîneraient d'ailleurs plus de complication dans les expressions analytiques. C'est aux géomètres à décider laquelle des deux méthodes mérite la préférence, soit pour l'usage des Tables, soit pour les expressions introduites dans le calcul, où j'ai eu soin d'éviter absolument toute figure \& toute construction géométrique. ${ }^{71}$

Le jugement que Legendre porte sur le résultat de Landen dans sa formulation originale ne laisse guère de doute sur le fait qu'aux yeux du mathématicien français, la forme de la transformation liant les intégrales des arcs d'ellipses n'y était pas encore explicitement dégagée comme telle, puisque précisément Legendre présente le fait de recourir à deux arcs d'ellipses "dont les amplitudes et les excentricités [i.e. les modules] sont différentes" comme une faiblesse de la méthode de Landen qui rend le calcul incommode et le complique au lieu de le faciliter. La démarche de Legendre consiste alors en partant de son propre théorème de rectification, à chercher à exprimer la dérivée partielle $\frac{d E}{d c}$ en fonction de deux arcs d'ellipses appartenant à deux ellipses différentes mais liées entre elles par une loi. Il retrouve ainsi le théorème de Landen en mettant toutefois en relief la transformation qui n'était jusqu'alors qu'implicite dans la construction géométrique.

L'objet que je me propose maintenant, est de démontrer le théorème de M. Landen, en le déduisant de mes formules, \& d'ajouter quelques propositions à celle de ce savant géomètre. En combinant son théorème avec une équation aux différences partielles, trouvée dans le Mémoire précédent, j'en ai tiré cette conséquence, que dans une infinité d'ellipses, dont les excentricités varient suivant une loi donnée, depuis le cercle jusqu'à la ligne droite, la rectification définie de deux de ces ellipses, c'est-à-dire leur longueur totale, donnera immédiatement celle de toutes les autres. (...)

La rectification indéfinie réussit également dans la même suite d'ellipses, \& nous ferons voir qu'ayant choisi à volonté deux ellipses dans cette suite, on peut par le moyen de leurs arcs déterminer ceux de toutes les ellipses de la même suite. ${ }^{72}$

Legendre considère ainsi une famille d'ellipses de semi-axe transverse égal à l'unité mais de semi-axe conjugué $b$ et d'excentricité $c=\sqrt{1-b^{2}}$ variables (cf. Fig. 5), puis étudie les fonctions $E(c, \phi)$ et $F(c, \phi)$ correspondant aux arcs de ces ellipses mesurés respectivement depuis le petit et le grand axe. En faisant varier continûment le paramètre $c$, on obtient une infinité d'ellipses comprises entre le cercle (pour une excentricité nulle) et la droite (pour une excentricité $c=1$ ), sur chacune desquelles on détermine une infinité d'arcs en faisant varier continûment l'amplitude

\footnotetext{
${ }^{71}[47$, p. $644-5]$.

$72[47$, p. $645-6]$.
} 
$\phi$. Il est alors naturel que ce point de vue de la variation continue conduise à accorder un rôle de premier plan au coefficient aux différences partielles $\frac{d E}{d c}$ pour chaque valeur de l'arc $E(c, \phi)$.

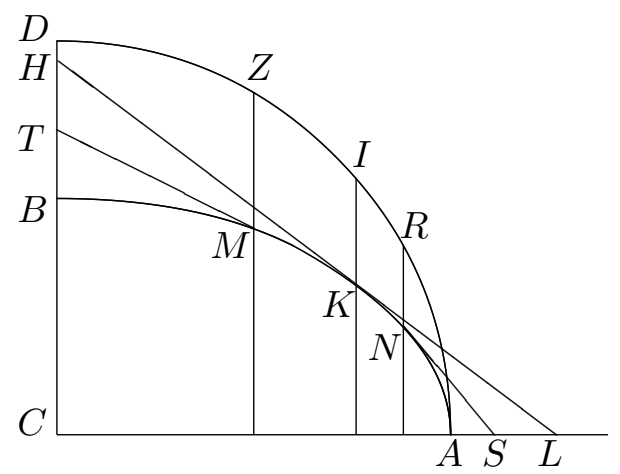

FIG. 8 - Le lemme de Fagnano (1716)

La réinterprétation par Legendre du théorème de rectification de Landen se fonde sur l'expression de ce coefficient $\frac{d E}{d c}$ pour des valeurs déterminées de $c$ et de $\phi$, au moyen de deux arcs $E$ et $E^{\prime}$ que l'on détermine sur des ellipses différentes mais dont les modules et les amplitudes sont liées par une loi. Pour obtenir ce résultat décisif, Legendre s'appuie sur un théorème de Fagnano (1716) portant sur les arcs d'ellipse dont la différence est assignable en ligne droite ${ }^{73}$, qu'il avait exploité dans le premier mémoire ([46, §III, p. 620-621]) dans la perspective de la constitution de tables numériques. En reprenant essentiellement l'idée directrice de Fagnano, Legendre montre que dans une ellipse de semi-grand axe égal à l'unité et de semi-axe conjugué égal à $b=\sqrt{1-c^{2}}$ (cf. Fig. 8), la différence entre les arcs $B M$ et $A N$ comptés respectivement à partir du petit axe et du grand axe et correspondant respectivement aux arcs de cercle $D Z=\phi, A R=\psi$, est égale à une quantité algébrique

$$
E(c, \phi)-F(c, \psi)=\frac{c^{2} \sin \phi \cos \phi}{\sqrt{1-c^{2} \sin ^{2} \phi}},
$$

pour autant que leurs amplitudes respectives $\phi, \psi$ sont liées par la relation $\tan \psi=b \tan \phi$. La preuve qu'il en donne ne consiste alors qu'en une simple vérification par le calcul. En différenciant la quantité algébrique exprimant la différence des arcs

$$
c^{2} V=\frac{c^{2} \sin \phi \cos \phi}{\sqrt{1-c^{2} \sin ^{2} \phi}}
$$

on obtiendrait par une série de transformations qui sont suggérées par le but à atteindre ${ }^{74}$

$$
c^{2} d V=d \phi \sqrt{1-c^{2} \sin ^{2} \phi}-\frac{\left(1-c^{2}\right) d \phi}{\left(1-c^{2} \sin ^{2} \phi\right)^{\frac{3}{2}}} .
$$

Mais la relation entre les amplitudes $\tan \psi=b \tan \phi$ permet de transformer l'expression fractionnaire ci-dessus de façon à faire apparaître un élément d'arc elliptique

$$
\frac{b^{2} d \phi}{\left(1-c^{2} \sin ^{2} \phi\right)^{\frac{3}{2}}}=d \psi \sqrt{1-c^{2} \cos ^{2} \psi}
$$

\footnotetext{
${ }^{73}$ Legendre ne donne aucune indication concernant le mémoire de Fagnano auquel il se réfère, mais il est clair qu'il s'agit du mémoire de 1716 sur les arcs elliptiques, hyperboliques et cyclö̈daux ([20]).

${ }^{74}$ Legendre omet les étapes intermédiaires du calcul et ne donne que le résultat.
} 
d'où l'on tire

$$
c^{2} d V=d \phi \sqrt{1-c^{2} \sin ^{2} \phi}-d \psi \sqrt{1-c^{2} \cos ^{2} \psi}
$$

dont l'intégration donne le résultat souhaité. Legendre montre en outre que la différence des arcs est égale à la différence des tangentes

$$
\operatorname{arc} B M-\operatorname{arc} A N=M T-N S,
$$

de sorte qu'en faisant tendre l'un vers l'autre les points $Z$ et $R$ tels que les $\operatorname{arcs} D Z=\phi$ et $A R=\psi$ qu'ils déterminent vérifient la relation $\tan \psi=b \tan \phi$, ils se rencontrent en un point $I$ qui détermine sur l'ellipse un point $K$ tel que la différence des $\operatorname{arcs} B K, K A$ est égale à la différence des demi-axes $C A, C B$, soit $1-b$, laquelle représente aussi le maximum de la différence des arcs, par conséquent, dans le cas général, on a l'inégalité suivante

$$
\frac{c^{2} \sin \phi \cos \phi}{\sqrt{1-c^{2} \sin ^{2} \phi}} \leq 1-b
$$

En se fondant sur cette relation, Legendre parvient alors dans son second mémoire à exprimer le coefficient aux différences partielles $\frac{d E}{d c}$ associé à l'arc mesuré sur une première ellipse en fonction de deux autres arcs déterminés sur une seconde ellipse, liée à la première par une loi spécifique. Dans ce but, Legendre considère d'abord une ellipse auxiliaire de demi-axe transverse $C A=1$, de demi-axe conjugué $C B^{\prime}=b^{\prime}$ et d'excentricité $c^{\prime}$ (cf. Fig. 9). Sur cette seconde ellipse, il considère deux $\operatorname{arcs} B^{\prime} M^{\prime}$ et $A N^{\prime}$ qui correspondent respectivement aux valeurs des arcs de cercle $\phi^{\prime}=D Z$ et $\psi^{\prime}=A R$ tels que $\tan \psi^{\prime}=b \tan \phi^{\prime}$.

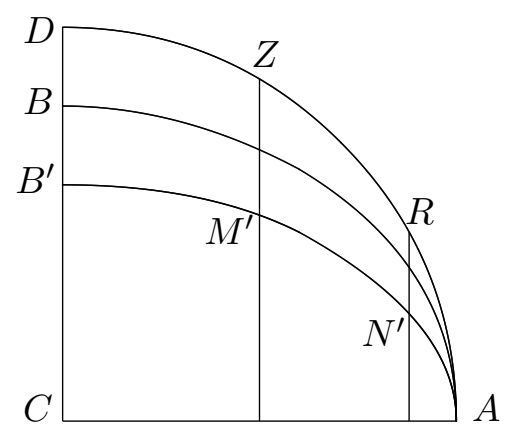

FIG. 9 - Les deux ellipses liées par la loi $c=\frac{1-b^{\prime}}{1+b^{\prime}}$

Comme nous venons de le voir, la différence de ces deux arcs d'ellipse est égale à une quantité algébrique majorée par $1-b^{\prime} \leq 1$. L'idée de Legendre consiste alors à introduire le sinus d'un troisième angle $\phi$ de manière à substituer à l'inégalité précédente $(I)$, l'égalité

$$
\frac{c^{\prime 2} \sin \phi^{\prime} \cos \phi^{\prime}}{\sqrt{1-c^{\prime 2} \sin ^{2} \phi^{\prime}}}=\left(1-b^{\prime}\right) \sin \phi
$$

qui permette d'exprimer $\sin \phi^{\prime}$ en fonction de $\sin \phi$. En posant le paramètre $c=\frac{1-b^{\prime}}{1+b^{\prime}}$, Legendre en tire une équation quadratique en $X=\sin ^{2} \phi^{\prime}$,

$$
X^{2}-\left(1+c \sin ^{2} \phi\right) \cdot X+\frac{c}{1-b^{2}} \sin ^{2} \phi=0,
$$


dont la résolution donne les valeurs suivantes ${ }^{75}$

$$
2 \sin ^{2} \phi^{\prime}=1+c \sin ^{2} \phi \pm \cos \phi \sqrt{1-c^{2} \sin ^{2} \phi}
$$

Par ailleurs, partant de la différentielle représentant l'élément d'arc $d E^{\prime}=d \phi^{\prime} \sqrt{1-c^{\prime 2} \sin ^{2} \phi^{\prime}}$, on peut remplacer le radical par sa valeur donnée par l'équation $(I I)$ et réexprimer les paramètres ${ }^{76}$ de façon à obtenir

$$
d E^{\prime}=\frac{2 \sin \phi^{\prime} \cos \phi^{\prime} d \phi^{\prime}}{(1+c) \sin \phi}
$$

et par suite

$$
2(1+c) d E^{\prime}=\frac{4 \sin \phi^{\prime} \cos \phi^{\prime} d \phi^{\prime}}{\sin \phi}
$$

que Legendre parvient à intégrer en rapportant le membre de droite à l'arc d'ellipse $E(c, \phi)$ et à son coefficient aux différences partielles $\frac{d E}{d c}$. Partant de l'expression pour $2 \sin ^{2} \phi^{\prime}$ (cf. $\left.(I I I)\right)$, on obtient en effet par différentiation et simplification ${ }^{77}$

$$
2(1+c) d E^{\prime}=2 c \cos \phi d \phi+2 \Delta d \phi-\frac{b^{2}}{\Delta} d \phi
$$

dont l'intégration donne la relation cherchée entre les arcs $E^{\prime}, E$ des deux ellipses ${ }^{78}$

$$
2(1+c) E^{\prime}=2 c \sin \phi+2 E-b^{2}\left(E-c \frac{d E}{d c}\right) .
$$

La conclusion qui peut en être tirée est alors la suivante :

l'arc indéfini $E^{\prime}$ peut se déterminer par le moyen de l'arc $E$ d'une autre ellipse, \& du coefficient aux différences partielles $\frac{d E}{d c}$. Il faut pour cela que l'excentricité de la seconde ellipse soit $c=\frac{1-b^{\prime}}{1+b^{\prime}}$, \& que les amplitudes $\phi$ et $\phi^{\prime} \operatorname{des} \operatorname{deux} \operatorname{arcs} E, E^{\prime}$ aient la relation comprise dans la l'équation $[(I I)$ ou $(I I I)]$.

Réciproquement, on peut se servir des deux $\operatorname{arcs} E \& E^{\prime}$ qui ont entre eux une si grande affinité, pour éviter d'employer dans le calcul le coefficient $\frac{d E}{d c}$; ainsi dans tous les cas où l'on aura fait usage de ce coefficient, ou de l'intégrale $\int \frac{d \phi}{\Delta}$ qui le renferme, on pourra mettre à leur place les valeurs $(\ldots)$ exprimées par deux arcs d'ellipse. ${ }^{79}$

Legendre est enfin en mesure de retrouver le théorème de Landen à partir de son propre théorème de rectification par un simple jeu de substitutions ${ }^{80}$, ce qui l'amène du même coup à produire une interprétation du résultat de son prédécesseur en mettant en relief la transformation qui y était seulement implicite.

\footnotetext{
${ }^{75}$ Comme précédemment, le calcul n'est qu'esquissé par Legendre, mais les étapes intermédiaires peuvent être facilement suppléées à partir de la forme qu'il choisit de donner au résultat.

${ }^{76}$ Plus précisément, on utilise la relation $\frac{1+c}{2}=\frac{1-b^{\prime}}{c^{\prime 2}}$ qui se déduit immédiatement de la stipulation $c=\frac{1-b^{\prime}}{1+b^{\prime}}$, pour autant que l'excentricité $c^{\prime}$ et le demi-axe conjugué $b^{\prime}$ de l'ellipse sont liés par la relation $c^{\prime 2}=1-b^{\prime 2}$.

${ }^{77}$ L'équation quadratique donne deux valeurs pour $\sin ^{2} \phi^{\prime}$ selon le choix du signe devant le radical. En différentiant la valeur la plus petite, on trouverait en effet une expression du numérateur $4 \sin \phi^{\prime} \cos \phi^{\prime} d \phi^{\prime}$ en fonction de $\sin \phi$ et de $\cos \phi$, d'où résulte immédiatement l'expression souhaitée de $2(1+c) d E^{\prime}$.

${ }^{78}$ Legendre utilise en particulier la relation $\int \frac{d \phi}{\Delta}=E-c \frac{d E}{d c}$, obtenue plus tôt ([46, p. 628-629]) dans le contexte d'une étude générale des méthodes de réduction aux arcs d'ellipse des expressions du type $\int \Delta^{2 m+1} d \phi, \int \frac{d \phi}{\Delta^{2 m+1}}$. Cf. supra, $\S 3.1$.

${ }^{79}[47$, p. $651-2]$.

${ }^{80} \mathrm{Il}$ suffit en effet, dans l'expression de l'arc hyperbolique $F M^{\prime}$ exposée plus haut (cf. $\S 3.2$ ), de substituer au coefficient aux différences partielles sa valeur en fonction des $\operatorname{arcs} E, E^{\prime}$, soit $b^{2} c \frac{d E}{d c}=2(1+c) E^{\prime}-\left(1+c^{2}\right) E-2 c \sin \phi$.
} 
Maintenant il est clair que l'arc d'hyperbole peut s'exprimer par les deux arcs d'ellipse $E, E^{\prime}, \&$ qu'on a

$$
H=\Delta \tan \phi+2 c \sin \phi+E-2(1+c) E^{\prime},
$$

c'est la belle proposition dont M. Landen a enrichi la géométrie. ${ }^{81}$

Le pas suivant consiste à former une suite d'ellipses successivement liées les unes aux autres d'après la même loi que celle qui liait les deux ellipses du théorème de rectification. Partant d'une ellipse de demi-grand axe égal à l'unité, de demi-axe conjugué $b^{\prime}=\sqrt{1-c^{\prime 2}}$ et d'excentricité $c^{\prime}$, Legendre en introduisait une seconde de demi-grand axe égal à l'unité, de demi-axe conjugué $b$ et d'excentricité $c$, telle que l'on ait $c=\frac{1-b^{\prime}}{1+b^{\prime}}$. En utilisant cette relation et la relation inverse ${ }^{82}$, il obtient alors par itération une suite d'excentricités croissante dans un sens

$$
c^{\prime}=\frac{2 \sqrt{c}}{1+c}, \quad c^{\prime \prime}=\frac{2 \sqrt{c^{\prime}}}{1+c^{\prime}}, \quad c^{\prime \prime \prime}=\frac{2 \sqrt{c^{\prime \prime}}}{1+c^{\prime \prime}}, \quad \ldots
$$

et décroissante dans l'autre

$$
c^{\circ}=\frac{1-b}{1+b}, \quad c^{\circ}=\frac{1-b^{\circ}}{1+b^{\circ}}, \quad c^{\circ 00}=\frac{1-b^{\circ \circ}}{1+b^{\circ \circ}}, \quad \ldots
$$

de sorte que la suite complète des excentricités ait d'un côté zéro pour limite et de l'autre l'unité

$$
0 \quad \ldots \quad c^{\circ 00}, c^{\circ \circ}, c^{\circ}, c, c^{\prime}, c^{\prime \prime}, c^{\prime \prime \prime}, \quad \ldots \quad 1
$$

Partant d'une relation remarquable liant entre elles les intégrales complètes $E_{1}, E_{1}^{\prime}$ et $E_{1}^{\prime \prime}$, représentant les quarts de trois ellipses consécutives de cette suite, d'excentricités respectives $c$, $c^{\prime}, c^{\prime \prime}$, Legendre en tire la proposition générale que la rectification de l'une quelconque des ellipses de cette suite peut se réduire à celle de deux autres prises arbitrairement dans cette même suite. En les choisissant en particulier aussi peu différentes du cercle qu'on voudra, on pourra ainsi mettre la rapidité de la convergence à profit, en parvenant en peu d'étapes à des approximations suffisantes. En passant aux intégrales indéfinies, Legendre généralise ensuite cette méthode aux arcs d'ellipse arbitrairement choisis à cette différence près que l'équation exprimant la relation entre les arcs pris sur des ellipses différentes comporte désormais des termes trigonométriques supplémentaires.

\section{De la classification des transcendantes aux échelles de mo- dules}

\subsection{Les formules d'approximation et la transformation de Landen}

Dans son second mémoire sur les intégrations par arcs d'ellipse, Legendre souligne la concordance entre ses propres résultats et ceux que Lagrange avait obtenus par une méthode différente. Dans un mémoire présenté à l'Académie de Turin, Sur une nouvelle méthode de calcul intégral ${ }^{83}$, Lagrange parvient en effet, à partir de principes très étrangers à ceux qui guident Legendre, à des formules d'approximation analogues pour les différentielles irrationnelles qui ne sont pas ordinairement intégrables par les logarithmes et les arcs de cercle.

\footnotetext{
${ }^{81}[47$, p. 652$]$.

${ }^{82}$ Les deux expressions sont en effet équivalentes. Partant de l'une d'entre elles, on forme une équation quadratique dont la solution conduit à l'autre.

${ }^{83}$ Bien que portant la mention d'une publication dans les Mémoires de l'Académie royale des sciences de Turin de 1785, le mémoire de Lagrange ne paraît effectivement que l'année suivante, comme le précise René Taton ([60, p. 20]).
} 
Je ne puis m'empêcher [écrit ainsi Legendre] de remarquer à ce sujet l'accord singulier de deux résultats obtenus par des méthodes totalement différentes. M. de la Grange a considéré dans les Mémoires de Turin, tome $V$, les différencielles de l'espèce de celles qui s'intègrent par les arcs des sections coniques; dans certains cas ces différentielles s'intégreraient exactement par les moyens ordinaires, c'est-à-dire, par les arcs de cercle \& les logarithmes; il faudrait pour cela une certaine relation entre les constantes. Or, dans tout autre cas M. de la Grange parvient par des substitutions successives, à approcher de plus en plus du point où une telle relation entre les coéfficiens rendrait l'intégrale possible. Il est clair que le résultat de cette méthode s'accorde parfaitement avec le nôtre. [47, p. 647]

Lagrange considère les différentielles irrationnelles de la forme $\frac{P d x}{R}$ où $P$ est une fonction rationnelle de $x$ et $R$ la racine carrée d'un polynôme du quatrième degré en $x$. Dans la mesure où la méthode qu'il propose ne s'applique que dans le cas de différentielles ne contenant pas de puissances impaires de $x$, il commence par les faire disparaître. Par une série de substitutions algébriques dont il s'assure qu'elles préservent la condition de réalité des variables ${ }^{84}$, il se ramène à une différentielle de la forme $\frac{Q d x}{\sqrt{\alpha+\beta x^{2}+\gamma x^{4}}}$, où $Q$ est une fonction rationnelle de $x^{2}$. Mais comme la méthode requiert en outre que le trinôme sous le radical soit résoluble en deux binômes quadratiques sans terme de puissance impaire, il cherche à obtenir, par une nouvelle transformation, une différentielle de la forme $\frac{N d x}{\sqrt{\left(a+b x^{2}\right)\left(m+n x^{2}\right)}}$, où $N$ est une fonction rationnelle de $x^{2}$ et $a, b, m, n$ sont des coefficients réels. Parvenu à ce point, Lagrange est en mesure d'appliquer la méthode nouvelle dont il définit l'esprit en ces termes.

Ainsi, toute la difficulté ne consiste qu'à trouver l'intégrale de cette dernière différentielle. Quant à l'intégrale exacte, elle paraît impossible en général; du moins l'analyse connue ne fournit aucun moyen pour l'obtenir. Mais il y a deux cas où elle se présente d'elle-même : le premier est celui où l'un des coefficients $b, n$ est nul, l'autre celui où $\frac{b}{a}=\frac{n}{m}$; dans ce dernier l'irrationnalité disparaît, et dans le premier il ne reste que l'irrationnalité relative à la quadrature du cercle ou de l'hyperbole, et qu'on peut toujours faire disparaître par les méthodes connues. Si donc la proposée n'est pas exactement dans l'un de ces deux cas, mais seulement dans un cas très-voisin de l'un d'eux, c'est-à-dire si l'une des quantités $\frac{b}{a}, \frac{n}{m}$ est très-petite, ou si elles sont à très-peu près égales, on pourra alors, au défaut d'une intégrale exacte, en avoir une très-approchée par le moyen des séries, et d'autant plus approchée que la quantité supposée très-petite le sera davantage, en disposant la série relativement aux puissances ascendantes de cette quantité. La méthode que je vais exposer a pour objet de ramener à cet état toute différentielle de la forme proposée, quels que soient les coefficients $a, b, m, n .[39$, p. 261]

Lagrange transforme d'abord la différentielle proposée $\frac{N d x}{\sqrt{\left(a+b x^{2}\right)\left(m+n x^{2}\right)}}$ en une autre de la forme $L d y+\frac{M d y}{\sqrt{\left(1 \pm p^{2} y^{2}\right)\left(1 \pm q^{2} y^{2}\right)}}$ où $L$ et $M$ sont des fonctions rationnelles de $y^{2}, p$ et $q$ des quantités réelles positives telles que $p>q$, et où le radical $R=\sqrt{\left(1 \pm p^{2} y^{2}\right)\left(1 \pm q^{2} y^{2}\right)}$ est nécessairement réel, puis il remarque qu' "une fois parvenu à une différentielle de cette dernière forme, il n'y a plus qu'à continuer et répéter les substitutions et les transformations que nous venons d'enseigner." 85 Par la transformation $y^{\prime}=\frac{y R}{1 \pm q^{2} y^{2}}$, la différentielle $\frac{M d y}{R}$ se change en $L^{\prime} d y^{\prime}+\frac{M^{\prime} d y^{\prime}}{R^{\prime}}$ où $L^{\prime}$ et $M^{\prime}$ seront des fonctions rationnelles de $y^{\prime 2}$ et $R^{\prime}=\sqrt{\left(1 \pm p^{\prime 2} y^{\prime 2}\right)\left(1 \pm q^{\prime 2} y^{\prime 2}\right)}$, avec

$$
p^{\prime}=p+\sqrt{p^{2}-q^{2}}, \quad q^{\prime}=p-\sqrt{p^{2}-q^{2}}
$$

\footnotetext{
${ }^{84}$ Cf. [39, p. 258] : "Cette condition de la réalité des variables introduites par des substitutions n'est pas nécessaire lorsqu'il s'agit d'intégrales exactes et absolues, parce qu'on a des moyens de faire disparaître ensuite les imaginaires; mais elle devient indispensable dans les intégrations approchées, car on ne peut bien juger de la convergence d'une série, à moins que tous ses termes ne soient réels et évalués en nombres."

${ }^{85}[39$, p. 264].
} 
ou encore, dans le sens inverse,

$$
p=\frac{p^{\prime}+q^{\prime}}{2}, \quad q=\sqrt{p^{\prime} q^{\prime}},
$$

qui correspondent respectivement, comme Lagrange le souligne ${ }^{86}$, aux moyennes arithmétique et géométrique. Si $p$ et $q$ sont des quantités positives, alors $p^{\prime}$ et $q^{\prime}$ le seront aussi, de sorte que les quantités $p, p^{\prime}, p^{\prime \prime}, \ldots$ forment une suite croissante à l'infini, et les quantités $q, q^{\prime}, q^{\prime \prime}, \ldots$ une suite correspondante décroissante qui tend vers zéro. Lagrange remarque alors que ces deux suites sont divergentes l'une par rapport à l'autre, mais qu'elles deviennent convergentes "si on les continue en arrière ainsi $\ldots, " p, p, p, \ldots " q, q, q "{ }^{\prime \prime}$. Dans ce dernier cas, les deux suites convergent vers la moyenne arithmético-géométrique. De cette manière, la différentielle à intégrer $\frac{N d x}{\sqrt{\left(a+b x^{2}\right)\left(m+n x^{2}\right)}}$ se transformera en cette autre

$$
L d y+L^{\prime} d y^{\prime}+L^{\prime \prime} d y^{\prime \prime}+\ldots+\frac{Z d z}{\sqrt{\left(1 \pm r^{2} z^{2}\right)\left(1 \pm s^{2} z^{2}\right)}},
$$

où l'on pourra prendre $r$ et $s$ aussi loin que l'on voudra dans les suites correspondantes, soit dans le sens où elles divergent soit dans celui où elles convergent, de sorte qu'on ait respectivement $s$ aussi proche de zéro, et $r$ et $s$ aussi proches l'un de l'autre qu'on le souhaite. Chacune des deux formules d'approximation conduit alors à une expression algébrique ou intégrable par les logarithmes et les arcs de cercle.

Dans la deuxième partie de son mémoire, Lagrange applique cette méthode générale à la rectification des arcs elliptiques et hyperboliques. Mais en appliquant les transformations successives ainsi prescrites à la différentielle correspondant à l'élément d'arc d'ellipse, il est amené à considérer une série de différentielles irrationnelles qui ne correspondent plus à des ellipses comme c'est le cas dans les formules d'approximation de Legendre. C'est une première différence significative, mais ce n'est pas la seule.

Contrairement à Legendre dont nous avons vu plus haut qu'il ne devait prendre connaissance des travaux de Landen qu'entre le premier et le second mémoire sur les méthodes d'intégration par arcs d'ellipse, mais qu'il les lie ensuite étroitement à ses propres recherches, Lagrange de son côté fait certes beaucoup plus tôt référence, de manière explicite, quoique furtive, dans sa correspondance avec Condorcet ${ }^{88}$, au théorème de rectification de l'hyperbole dont il semble avoir alors été intrigué, mais ne semble pas y rapporter sa méthode générale d'intégration. Aucun élément ne permet en effet d'inférer que cette lecture antérieure ait pu l'inciter d'une quelconque façon à élaborer la méthode générale exposée dans le mémoire de Turin. S'il est acquis, comme on le reconnaît traditionnellement ${ }^{89}$, que l'algorithme de la moyenne arithméticogéométrique apparaît pour la première fois dans le mémoire de Lagrange, on peut en revanche légitimement douter que le mathématicien français ait lui-même regardé la transformation dont l'itération constitue la cheville ouvrière de sa méthode générale comme l'explicitation d'une transformation qui n'aurait été qu'implicite dans le résultat de Landen. Il n'est d'ailleurs nulle part fait mention du theorème de Landen dans le mémoire de Lagrange. Quoique l'absence de pièces documentaires ne nous permette guère de formuler que de simples conjectures sur la teneur probable des échanges entre Lagrange et Legendre au cours des mois qui séparent la

\footnotetext{
${ }^{86}$ Cf. [39, p. 267].

87 [39, p. 271].

${ }^{88} \mathrm{Cf}$. la lettre à Condorcet du 3 janvier 1777, [40, tome XIV, p. 41] : "J'ai vu, dans le dernier Volume des Transactions philosophiques, un théorème de M. Landen qui me paraît bien singulier. Il réduit la rectification des arcs elliptiques à celle des arcs hyperboliques. Je n'ai pas encore eu le temps d'examiner s'il n'y a pas de paralogisme dans la démonstration."L'index qui clôt le tome XIV des Euvres de Lagrange n'indique aucune autre référence à Landen dans tout le corpus.

${ }^{89}$ Cf. [23, p. 191], [10, p. 275], [32, p. 108].
} 
présentation par ce dernier de son premier mémoire devant l'Académie des sciences de Paris en 1786 et la publication du second mémoire, dans le même volume que le premier, en 1788, on peut néanmoins supposer que Lagrange a eu d'une manière ou d'une autre connaissance de ce premier mémoire, et qu'en y découvrant un théorème de rectification de l'hyperbole, il a pu prendre l'initiative de signaler à Legendre le résultat de Landen qu'il connaissait pour sa part depuis 1777. Bien qu'il fût lié à l'Académie de Paris au titre d'associé étranger depuis 1772, il ne devait quitter Berlin et s'installer dans la capitale française qu'à l'été 1787. Il n'a donc pas pu assister à la lecture du premier mémoire de Legendre. Néanmoins il est très probable que Lagrange ait suivi les travaux de Legendre dont il avait remarqué ${ }^{90}$ la valeur dès 1783 et dont nous savons en outre, par Delambre, que Lagrange devait lui être grandement redevable de s'être chargé de la révision des épreuves de sa Mécanique analytique que son auteur souhaitait faire imprimer à Paris ${ }^{91}$. Quoique nous ne sachions pas précisément quand ont débuté ces travaux de relecture, ils se sont vraisemblablement poursuivis après l'installation de Lagrange à Paris et ont sans doute, à en croire René Taton ${ }^{92}$, été l'occasion d'une collaboration suivie entre les deux hommes. Dans la période qui précède la publication de son second mémoire, Legendre a donc non seulement sous les yeux le mémoire de Lagrange Sur une nouvelle méthode de calcul intégral, mais il est en outre très vraisemblable qu'il ait pu s'en entretenir avec l'auteur. Que dans ces conditions, il ait eu à cœur de marquer la différence des méthodes, en dépit de la concordance des résultats, tendrait à témoigner davantage encore de l'originalité de l'interprétation qu'il produit en appliquant ses propres principes au théorème de Landen pour dégager la forme de la transformation à laquelle s'attache depuis le nom du mathématicien britannique.

\subsection{Les motivations algorithmiques de la classification des transcendantes}

Legendre voyait dans la forme achevée de sa théorie des fonctions elliptiques la réalisation des vœux qu'Euler avait formés pour développer la théorie des transcendantes grâce à l'assignation de caractères susceptibles d'algorithmes appropriés.

Il ne sera pas inutile pour l'histoire de la Science [note-t-il dès les premières pages de son traité] de faire remarquer ici que cette nouvelle branche d'analyse à laquelle l'Auteur a donné le nom de Théorie des fonctions elliptiques, est fondée en grande partie sur les bases établies dans le chapitre $\mathrm{V}$ concernant la forme la plus simple de ces fonctions et leur division en trois

\footnotetext{
${ }^{90}$ Cf. la lettre à Laplace du 15 septembre 1782, [40, tome XIV, p. 116] : "Connaissez-vous M. Legendre? Il vient de remporter notre prix sur la Balistique. Sa pièce m'a paru aussi bonne que le sujet peut le comporter, et elle annonce dans son auteur, s'il est jeune encore, des talents et des connaissances qui pourront le mener loin; je vous prie de lui dire la part que je prends à son succès." La réponse de Laplace du 10 février atteste de la date à laquelle Lagrange et Legendre entrent en relation l'un avec l'autre, [40, tome XIV, p. 121] : "J'ai fait part à M. Legendre des choses obligeantes que vous me marquez sur son compte; il y est infiniment sensible, et il m'a chargé de vous en témoigner toute sa reconnaissance; c'est un jeune homme d'un rare mérite, et qui est avantageusement connu de l'Académie par plusieurs excellents Mémoires dont j'ai été rapporteur." Une lettre ultérieure de Laplace à Lagrange, datée du 21 août 1783, témoigne alors du fait que des relations directes s'étaient établies entre Legendre et Lagrange, quoique les lettres nous fassent défaut, car c'est désormais Legendre qui sert d'intermédiaire entre Laplace et Lagrange, [40, tome XIV, p. 124] : "M. Legendre me dit que vous avez reçu l'exemplaire de mon Mémoire sur les comètes, et que vous vous proposez de m'envoyer quelques nouveaux Mémoires que vous faites maintenant imprimer. Je les attends avec la plus vive impatience."

${ }^{91}$ Cf. le témoignage de Delambre, [13, p.XXXIV] : "M. Legendre se dévoua tout entier à cette révision pénible, et s'en trouvait payé par le sentiment de vénération dont il était pénétré pour l'Auteur, et par les remerciements qu'il en reçut dans une lettre que j'ai eue entre les mains, et que M. Lagrange avait remplie des expressions de son estime et de sa reconnaissance."

${ }^{92}$ Cf. [61, p. 60] : "Si la suite des procès-verbaux des séances de l'Académie pour l'année 1787 atteste l'assiduité de Lagrange, elle ne mentionne aucune trace d'activité personnelle en son sein. Il ne pouvait, semble-t-il, se désintéresser complètement du sort de sa Méchanique analitique qui se trouvait alors en cours d'impression chez la veuve Desaint. Bien que Delambre ait affirmé que les épreuves de cet ouvrage ne furent corrigées que par Legendre, auquel Lagrange adressa une émouvante lettre de remerciement, malheureusement perdue, on peut penser que, sur bien des points, Legendre obtint l'aide et l'approbation de l'auteur de ce livre aujourd'hui classique."
} 
espèces ; d'où est résulté un système de nomenclature et de notation, propre à représenter ces fonctions dans les usages ordinaires de l'analyse, et à faciliter la recherche de leurs propriétés. Euler avait prévu qu'à l'aide d'une notation convenable, le calcul des arcs d'ellipse et autres transcendantes analogues, pourrait devenir d'un usage presque aussi général que celui des arcs de cercle et des logarithmes; mais si on excepte Landen, qui, par la découverte de son théorème, aurait pu s'ouvrir des routes nouvelles, personne ne s'est mis en devoir de réaliser la prédiction d'Euler. ${ }^{93}$

Dans ce passage du Traité de 1825, Legendre se réfère implicitement à l'un des mémoires d'Euler, mentionnés précédemment, qui se situent dans la lignée des recherches de Maclaurin et d'Alembert, le "De reductione formularum integralium ad rectificationem ellipsis ac hyperbola" ([18]), dans lequel Euler se propose d'introduire les arcs de sections coniques dans le domaine de l'Analyse au même titre que les transcendantes plus simples qui y ont déjà droit de cité, à savoir les logarithmes et arcs de cercle et leurs inverses. Tout comme on convient, pour les fonctions trigonométriques, de considérer le cercle de rayon unité, Euler fixe le demi-paramètre de la conique égal à l'unité de façon à obtenir tout le spectre des coniques en faisant varier un unique paramètre $a$ qui correspond au demi-grand axe ${ }^{94}$. Il introduit alors le symbole $\Pi x[a]$ pour désigner les longueurs d'arc et commence à développer un calcul dont il espère de grands progrès pour l'Analyse : “de tels signes fourniront une espèce de calcul dont j'ai résolu d'exposer ici comme les premiers éléments" 95. Mais à la différence de ce qu'accomplira par la suite Legendre, le choix des types d'intégrales est encore tributaire chez Euler des méthodes d'intégration par arcs d'ellipse et d'hyperbole et demeure de ce fait largement déterminé par leur signification géométrique ${ }^{96}$.

Les formules d'approximation fondées sur les suites d'ellipses successivement liées par une loi représentaient sans doute un pas important en direction d'un tel calcul des transcendantes, mais, comme nous l'avons dit plus haut, les arcs d'ellipse ne pouvaient suffire à résoudre les problèmes d'analyse qui dépendent d'une différentielle de la forme générale $\int \frac{P d x}{R}$ où $P$ est une fonction rationnelle de $x$ et $R$ la racine d'un polynôme du quatrième degré. Dans le Memoire sur les transcendantes elliptiques qu'il présente devant l'Académie de Paris en 1792 et publie l'année suivante, Legendre se propose donc d'étudier de manière systématique les transcendantes qui relèvent de cette forme, de les comparer entre elles, de les réduire aux formes les plus simples dont elles sont susceptibles, enfin d'obtenir pour la classe entière qu'elles constituent des formules d'approximation analogues à celles obtenues pour les arcs d'ellipse dans le mémoire précédent. Dans ce but, il utilise certaines méthodes de réduction des différentielles irrationnelles qu'il emprunte à D'Alembert et à Lagrange, mais qu'il combine d'une manière originale. Dans l'éloge historique qu'il fait du mathématicien français, Élie de Beaumont, secrétaire perpétuel de l'Académie des sciences, décrit la démarche de Legendre dans les termes suivants.

Reprenant dans sa forme algébrique la plus générale la différentielle déjà indiquée comme point de départ de ce genre de recherches [à savoir $\left.\int \frac{P d x}{R}\right]$, il la dégrossit avec une adresse infinie, met de côté toutes les parties qui s'intègrent soit par des quantités purement algébriques, soit par des arcs de cercle ou des logarithmes, et la réduit ainsi à sa quintessence, c'est-à-dire aux parties dont les intégrales sont les transcendantes d'un ordre supérieur. Transformant ensuite ce résidu au moyen des fonctions circulaires, il le réduit à une forme d'une merveilleuse simplicité qui ne contient que cinq quantités [c'est-à-dire $\left.H=\int \frac{A+B \sin ^{2} \phi}{1+n \sin ^{2} \phi} \frac{d \phi}{\Delta}\right]$ : un arc de

\footnotetext{
93 [50, Avertissement, p. vi et vii].

${ }^{94}$ Sur la démarche d'Euler dans ce mémoire, cf. [62, p. 836-837], [25, p. XLII].

${ }^{95}[18$, p. 258] : "Talia signa novam quandam calculi speciem suppeditabunt, cujus hic quasi prima elementa exponere constitui".

${ }^{96}$ Sur la différence entre la notation et l'ébauche de calcul d'Euler et la théorie de Legendre, cf. [25, p. XLII] : "Cependant, Euler ne construit pas de table numérique qui permettrait d'utiliser pratiquement ses résultats analytiques et, de plus, il conserve encore comme intégrales fondamentales celles qui correspondent à la mesure des arcs d'ellipse et d'hyperbole. Sur ces deux points, c'est Legendre qui va apporter les progrès décisifs."
} 
cercle désigné sous le nom d'amplitude, qui est nul au point où commence l'intégrale et se développe à mesure qu'elle s'étend; un module toujours réel et plus petit que l'unité qui, dans le cas où il s'agit d'une ellipse, en représente l'excentricité; un paramètre d'une grandeur quelconque, positif ou négatif, qui peut se réduire à zéro, mais auquel il serait inutile d'attribuer des valeurs imaginaires; enfin deux coefficients dont les valeurs indépendantes de tout le reste peuvent être quelconques pourvu qu'elles ne soient pas nulles simultanément. $[2$, p. 40]

Plus précisément, Legendre montre d'abord en recourant à une méthode de réduction reprise essentiellement de d'Alembert ${ }^{97}$ que l'intégrale $\int \frac{P d x}{R}$, peut se décomposer en trois parties principales, la première algébrique, la seconde de la forme $\int \frac{\left(A+B x+C x^{2}\right) d x}{R}$, et la troisième renfermant une ou plusieurs intégrales de la forme $\int \frac{d x}{(1+n x) R}$, où le coefficient $n$ peut être réel ou imaginaire. Cette méthode se fonde sur l'usage de formules de récurrence du type de celle qu'on obtient entre les intégrales de la forme $\Pi^{k}=\int \frac{x^{k} d x}{R}$ à partir du produit $x^{m-3} R$, par différentiation puis intégration. Par itération de telles formules de récurrence, on montre que $\Pi^{m}$ s'exprime alors comme combinaison linéaire des intégrales $\Pi^{0}$, $\Pi^{1}$ et $\Pi^{2}$, d'où l'on tire la réduction à la forme $\int \frac{\left(A+B x+C x^{2}\right) d x}{R}$ mentionnée ci-dessus, tandis que la réduction à l'autre forme $\int \frac{d x}{(1+n x) R}$ procède de formules de récurrence analogues pour les intégrales $\Gamma^{k}=\int \frac{d x}{(1+n x)^{k} R}$. Comme s'il prenait un nouveau départ, Legendre propose alors dans un second temps de "suivre une autre route pour parvenir à une connaissance plus précise des mêmes transcendantes" 98 . Il reprend et complète la méthode de Lagrange pour faire disparaître les puissances impaires de la variable sous le radical dans la différentielle $\frac{P d x}{R}$, en tirant pleinement parti d'une substitution suggérée, mais finalement écartée par Lagrange parce qu'elle ne permettait pas de garantir la réalité des variables que ce dernier jugeait cependant nécessaire, comme nous l'avons vu plus haut [vide supra, note 84], pour le calcul des valeurs approchées des intégrales ${ }^{99}$. À la différence de son prédécesseur, Legendre utilise toutefois cette réduction qu'il amende de façon à faire apparaître deux facteurs quadratiques réels sans terme de puissance impaire, mais il fait un pas supplémentaire décisif et ramène, par un changement de variable approprié, l'intégrale $\int \frac{P d x}{R}$ à la formule $\int \frac{Q d \phi}{\sqrt{1-c^{2} \sin ^{2} \phi}}$ dans laquelle $c$ est une quantité réelle comprise entre zéro et l'unité et $Q$ une fonction rationnelle paire de $\sin \phi$ qui contient $\sin \phi$ au même degré que $P$ contient $x$. En transposant ensuite la méthode de d'Alembert fondée sur les formules de récurrence à cette formule générale $\int \frac{Q d \phi}{\sqrt{1-c^{2} \sin ^{2} \phi}}$, il est alors en mesure de la ramener à (1) une partie algébrique, (2) une intégrale de la forme $\int\left(A+B \sin ^{2} \phi\right) \frac{d \phi}{\Delta}$ où $\Delta=\sqrt{1-c^{2} \sin ^{2} \phi}$, et (3) une ou plusieurs parties de la forme $\int \frac{N d \phi}{\left(1+n \sin ^{2} \phi\right) \Delta}$, où les coefficients $N$ et $n$ ont des valeurs quelconques, réelles ou imaginaires. Dans une dernière étape enfin, Legendre dégage par une induction judicieuse la formule générale $H=\int \frac{A+B \sin ^{2} \phi}{1+n \sin ^{2} \phi} \frac{d \phi}{\Delta}$ dont les deux formes principales ci-dessus auxquelles sa méthode de réduction aboutit, ne sont que des espèces ${ }^{100}$.

L'exigence d'étendre le calcul des transcendantes de la classe restreinte des arcs d'ellipse à la classe entière des intégrales elliptiques guide ainsi la démarche d'ensemble de Legendre en suggérant l'enchâssement des formes réduites dans une formule générale unique $\int \frac{Q d \phi}{\sqrt{1-c^{2} \sin ^{2} \phi}}$ qui puisse constituer le pendant de la formule analogue pour les arcs d'ellipse. Mais plus encore

\footnotetext{
${ }^{97}$ Christian Gilain note en effet que "cette méthode de Legendre [.. .] est, à des détails techniques près, exactement la même que celle de d'Alembert" [25, p. XLIV]. Sur cette méthode exposée dans les Recherches sur le calcul intégral de 1746, cf. l'analyse et le tableau d'ensemble qu'en donne Christian Gilain, [25, p. XXXVII-XXXIX].

98 [48, p. 8].

${ }^{99}$ Sans mentionner le nom de son prédécesseur dans ce contexte, Legendre [48, p. 9] reprend exactement la substitution à laquelle Lagrange [39, p. 258] fait allusion, à savoir $x=\frac{p+q y}{1+y}$, et montre qu'elle permet d'obtenir des facteurs quadratiques reels.

${ }^{100}$ Il suffit de prendre $n=0$ dans $H$ pour obtenir la première, et de prendre $B=0$ pour obtenir la seconde.
} 
la possibilité, ou l'impossibilité, d'exprimer les transcendantes de cette forme en termes d'arcs d'ellipse fonde leur classification en trois espèces distinctes. Dans la mesure où la fonction $H$ prise dans toute sa généralité ne peut pas se réduire à des arcs d'ellipse, il est en effet opportun, comme l'explique Legendre, de distinguer la formule $G=\int\left(A+B \sin ^{2} \phi\right) \frac{d \phi}{\Delta}$ comme une espèce indépendante à laquelle on arrive par spécification des paramètres de $H$ et qui présente la propriété de pouvoir être exprimée au moyen de deux arcs d'ellipse. De ce point de vue, la formule $G$ renferme comme cas particuliers aussi bien les arcs d'ellipse que les arcs d'hyperbole dont Legendre choisit de ne pas faire deux espèces distinctes, parce qu'ils peuvent indifféremment s'exprimer les uns par les autres. En revanche, c'est précisement pour la raison inverse que l'intégrale $\int \frac{d \phi}{\sqrt{1-c^{2} \sin ^{2} \phi}}$ se signale comme une formule "extrêmement remarquable" qui mérite d'être désignée au moyen d'un caractère particulier $F$.

La quantité $F$ peut bien s'exprimer par $E$, mais non pas $E$ par $F$, et encore moins $G$ par $F$; et c'est, ce me semble, une preuve certaine que la fonction $F$, considérée analytiquement, est moins composée que les arcs d'ellipse.[48, p. 19]

Dans le mémoire de 1793, Legendre distingue donc les trois espèce suivantes : (1) $F=\int \frac{d \phi}{\Delta}$, (2) $G=\int\left(A+B \sin ^{2} \phi\right) \frac{d \phi}{\Delta}$ qui regroupe les arcs d'ellipse et les arcs d'hyperbole, et enfin (3) la formule générale $H=\int \frac{A+B \sin ^{2} \phi}{1+n \sin ^{2} \phi} \frac{d \phi}{\Delta}$. Par la suite, dans les Exercices de 1811, comme dans le Traité de 1825, Legendre propose une classification plus fine

$$
\text { 1. } F=\int \frac{d \phi}{\Delta}, \quad \text { 2. } E=\int \Delta \phi, \quad \text { 3. } \Pi=\int \frac{d \phi}{\left(1+n \sin ^{2} \phi\right) \Delta},
$$

qui, comme le souligne Christian Gilain [25, p. XIV], correspond à une hiérarchisation des transcendantes selon la complexité croissante des formules d'addition. Dans le cas de la fonction $F$, la relation est la plus simple : $F(x)+F(y)=F(z)$ où $z$ est une fonction algébrique de $x$ et de $y$. Viennent ensuite les arcs d'ellipse pour lesquels le théorème d'addition fait intervenir un terme supplémentaire : $E(x)+E(y)=E(z)+f(x, y)$ où $f(x, y)$ est une fonction algébrique de $x$ et de $y$. Enfin la troisième espèce de transcendantes manifeste encore un degré de complexité de plus car on a alors $\Pi(x)+\Pi(y)=\Pi(z)+g(x, y)$ où $g(x, y)$ est une fonction transcendante de $x$ et de $y$.

\subsection{Des formules d'approximation étendues aux fonctions elliptiques de première espèce aux échelles de modules}

Selon la classification ainsi arrêtée des transcendantes elliptiques, la fonction $F$ apparaît plus simple d'un point analytique que les arcs d'ellipse $E$. Il était donc naturel que Legendre ait cherché à appliquer en premier lieu aux fonctions elliptiques de première espèce la méthode d'approximation élaborée précédemment pour les arcs d'ellipse, en étudiant la possibilité d'utiliser la même substitution pour former une famille de fonctions $F$ dont les modules seraient liés par une loi comme l'étaient les ellipses dans le mémoire sur les intégrations par arcs d'ellipse.

La méthode que nous allons suivre est la même que celle dont nous avons fait usage dans le volume cité de 1786 , pour ramener la rectification d'une ellipse donnée à celle de deux autres ellipses aussi peu différentes du cercle qu'on voudra. L'esprit de cette méthode consiste à ramener l'intégrale d'une différentielle affectée du radical $\sqrt{1-c^{2} \sin ^{2} \phi}$ à celle d'une différentielle semblable où le module $c$ soit plus petit que toute quantité donnée. [48, p. 38]

Legendre part donc de la substitution qui permettait d'exprimer un arc d'ellipse $E^{\prime}$ par le moyen de l'arc $E$ d'une autre ellipse et du coefficient aux différences partielles $\frac{d E}{d c}$ correspondant, 
de sorte que les deux ellipses soient liées entre elles par la loi $c=\frac{1-b^{\prime}}{1+b^{\prime}}$, à savoir la relation $(I I I)$ indiquée précédemment [vide supra, cf. §3.3] qu'il écrit désormais

$$
2 \sin ^{2} \phi=1+c^{\circ} \sin ^{2} \phi^{\circ}-\Delta^{\circ} \cos \phi^{\circ}
$$

où $\Delta^{\circ}=\sqrt{1-c^{\circ} \sin ^{2} \phi^{\circ}}$, puis montre par le calcul que cette substitution conduit, dans le cas des fonctions elliptiques de première espèce, à une relation remarquable dont il tirera les échelles de modules, à savoir

$$
F=\frac{1+c^{\circ}}{2} F^{\circ} .
$$

Plus précisément, le calcul de Legendre consiste à dériver les expressions respectives de $d \phi$ et de $\Delta=\sqrt{1-c^{2} \sin ^{2} \phi}$ en fonction de $\sin \phi^{\circ}$ et $\cos \phi^{\circ}$ à partir des deux seules équations parallèles suivantes

$$
\begin{aligned}
& 2 \sin ^{2} \phi=1+c^{\circ} \sin ^{2} \phi^{\circ}-\Delta^{\circ} \cos \phi^{\circ} \\
& 2 \cos ^{2} \phi=1-c^{\circ} \sin ^{2} \phi^{\circ}+\Delta^{\circ} \cos \phi^{\circ}
\end{aligned}
$$

On montre ${ }^{101}$ ainsi dans un premier temps que

$$
\Delta=\frac{\Delta^{\circ}+c^{\circ} \cos \phi^{\circ}}{1+c^{\circ}}
$$

Le calcul de $d \phi$ requiert ensuite, à titre de résultat auxiliaire, la relation suivante

$$
2 \sin \phi \cos \phi=\sin \phi^{\circ}\left(c^{\circ} \cos \phi^{\circ}+\Delta^{\circ}\right) .
$$

qui s'obtient en réorganisant les termes du produit de $2 \sin ^{2} \phi$ par $2 \cos ^{2} \phi$ dont les expressions en fonction de $c^{\circ}, \sin \phi^{\circ}$ et $\cos \phi^{\circ}$ sont connues (Equ. (42) et (43)). Par différenciation de l'équation

$$
2 \sin ^{2} \phi=1+c^{\circ} \sin ^{2} \phi^{\circ}-\Delta^{\circ} \cos \phi^{\circ},
$$

on obtient une expression de $4 \sin \phi \cos \phi . d \phi$ en fonction de $c^{\circ}, \sin \phi^{\circ}, \cos \phi^{\circ}$, d'où l'on tire immédiatement la valeur de $d \phi$

$$
d \phi=\frac{d \phi^{\circ}\left(c^{\circ} \cos \phi^{\circ}+\Delta^{\circ}\right)}{2 \Delta^{\circ}},
$$

puis

$$
\frac{d \phi}{\Delta}=\left(\frac{1+c^{\circ}}{2}\right) \frac{d \phi^{\circ}}{\Delta^{\circ}}
$$

dont l'intégration donne la relation entre les fonctions de première espèce $F=\frac{1+c^{\circ}}{2} F^{\circ}$.

La méthode d'approximation de Legendre consiste alors à former, à partir de la fonction $F$ que l'on se propose d'évaluer, une suite de fonctions de première espèce successivement liées par la même loi, de sorte qu'on puisse exprimer la fonction $F$ de départ au moyen d'une fonction quelconque de la suite dont le module peut être pris de plus en plus petit

$$
\begin{array}{r}
F=\frac{1+c^{\circ}}{2} F^{\circ}, \\
F=\frac{1+c^{\circ}}{2} \cdot \frac{1+c^{\circ \circ}}{2} F^{\circ \circ}, \\
F=\frac{1+c^{\circ}}{2} \cdot \frac{1+c^{\circ}}{2} \cdot \frac{1+c^{\circ \circ}}{2} F^{\circ \circ}, \\
\text { etc. }
\end{array}
$$

\footnotetext{
${ }^{101}$ Legendre ne donne que le résultat mais les étapes intermédiaires du calcul ne présentent aucune difficulté. Il suffit de développer $\Delta^{2}$ en remplaçant $c^{2}$ et $\sin ^{2} \phi$ par leurs valeurs respectives de façon à faire apparaître la formule du carré du binôme au numérateur.
} 
Lorsque le module $c$ tend vers zéro, $\Delta$ tend vers l'unité et la valeur de la fonction $F=\int \frac{d \phi}{\Delta}$ tend vers la valeur de l'angle $\phi$. Legendre considère ainsi une suite de modules décroissants tendant vers zéro $c, c^{\circ}, c^{\circ \circ}, c^{\circ 00}, \ldots$ et parallèlement la suite des amplitudes correspondantes $\phi, \phi^{\circ}, \phi^{\circ \circ}$, $\phi^{\circ 00}, \ldots$ dont il montre qu'elles sont successivement liées par la formule

$$
\tan \left(\phi^{\circ}-\phi\right)=b \tan \phi,
$$

ou par la série correspondante

$$
\phi^{\circ}=2 \phi-c^{\circ} \sin 2 \phi+\frac{1}{2} c^{\circ 2} \sin 4 \phi-\frac{1}{3} c^{\circ 3} \sin 6 \phi+\ldots
$$

En posant $\Phi$ la limite des angles $\phi, \frac{\phi^{\circ}}{2}, \frac{\phi^{\circ 0}}{4}, \frac{\phi^{\circ 00}}{8}$, etc., on obtient, pour la fonction de départ, l'expression suivante

$$
F=\Phi\left(1+c^{\circ}\right)\left(1+c^{\circ \circ}\right)\left(1+c^{\circ \circ 0}\right) \ldots
$$

qui en permet le calcul approché, ou de manière encore plus prompte, grâce aux tables de logarithme, sous la forme équivalente

$$
F=\Phi\left(\frac{2 \sqrt{c^{\circ}}}{c}\right)\left(\frac{2 \sqrt{c^{\circ \circ}}}{c^{\circ}}\right)\left(\frac{2 \sqrt{c^{\circ \circ 0}}}{c^{\circ \circ}}\right) \ldots
$$

En renversant l'ordre chronologique dans lequel les méthodes d'approximation ont été effectivement élaborées, Legendre suit, dès le mémoire de 1793, l'ordre logique dicté par la classification des transcendantes. La méthode d'approximation au moyen des échelles de modules n'est appliquée aux fonctions elliptiques de seconde espèce ${ }^{102}$ qu'après les fonctions $F$, alors même que l'idée de la méthode provient du calcul des arcs d'ellipse qui en sont un cas particulier remarquable.

Dans les Exercices de 1811 comme dans le Traité de 1825, Legendre devait mettre davantage en valeur les échelles de modules en choisissant de les exposer pour elles mêmes, conformément à l'exigence d'une mise en ordre systématique des transcendantes elliptiques, avant de montrer l'usage qui peut en être fait dans le contexte des méthodes d'approximation dont elles tirent précisément leur origine. La transformation de Landen y apparaît en outre sous une forme sensiblement différente, quoique parfaitement équivalente ${ }^{103}$. On pose en effet que si les amplitudes de deux fonctions elliptiques de première espèce dont les modules vérifient la relation $c^{\prime}=\frac{2 \sqrt{c}}{1+c}$ sont liées entre elles par la relation canonique suivante

$$
\sin \left(2 \phi^{\prime}-\phi\right)=c \sin \phi,
$$

alors les intégrales elliptiques correspondantes sont telles que $F\left(c^{\prime}, \phi^{\prime}\right)=\frac{1+c}{2} F(c, \phi)$. Legendre perfectionne plus encore la mise en ordre systématique des transcendantes, entre l'édition de 1811 et celle de 1825, grâce à la découverte d'une autre transformation remarquable qui lui permet d'élaborer une seconde échelle de modules ${ }^{104}$ distincte de la première et qui la complète de manière à former "une sorte de damier analytique, dont les cases correspondent aux transformations infiniment multipliées que peut subit la plus simple des fonctions elliptiques, sans cesser d'être semblable à elle-même" 105 . La théorie des fonctions elliptiques culminait ainsi dans une présentation synoptique offrant une vue unifiée des méthodes de Legendre.

\footnotetext{
${ }^{102}$ Aux fonctions $G=\int\left(A+B \sin ^{2} \phi\right) \frac{d \phi}{\Delta}$ dans le mémoire de 1793 , puis ensuite aux fonctions $E=\int \Delta d \phi$ dans les traités de 1811 et de 1825 .

${ }^{103}$ Les formulations de la transformation de Landen de 1793 et de 1811 sont équivalentes. Pour des modules liés par la relation $c^{\prime}=\frac{2 \sqrt{c}}{1+c}$, les Mémoires en donnent une version directement issue de la rectification de l'hyperbole $2 \sin ^{2} \phi^{\prime}=1+c \sin ^{2} \phi-\sqrt{1-c^{2} \sin ^{2} \phi} \cdot \cos \phi$, tandis que les Exercices privilégient la relation $\sin \left(2 \phi^{\prime}-\phi\right)=c \sin \phi$. Mais l'on passe sans difficulté de l'une à l'autre. Il suffit de développer $\sin \left(2 \phi^{\prime}-\phi\right)$ et de remplacer $\sin \phi^{\prime}$ et $\cos \phi^{\prime}$ par les valeurs données par la première relation pour obtenir la seconde.

104 [50, chap. XXXI].

105 [50, Avertissement, p. v-vi]. Sur le rôle régulateur de la fonction de première espèce dans la théorie du damier analytique de Legendre, cf. [59].
} 


\section{Conclusion}

Dans la Remarque Générale qui suit l'exposition de la première échelle de modules, Legendre s'interroge sur les différents chemins qui auraient pu mener ses prédécesseurs à cette découverte et le seul mérite qu'il revendique pour lui-même est d'avoir su tirer le système entier de la comparaison des modules de ce qu'il nomme la transformation de Landen.

Mais beaucoup d'autres substitutions [écrit-il] peuvent conduire à de semblables résultats, et quand on considère combien de transformations analytiques ont été employées par Maclaurin et d'Alembert, dans leurs recherches sur les intégrales qui peuvent être exprimées par des arcs de sections coniques, on a lieu de s'étonner que la transformation qui met en évidence les propriétés nombreuses de l'échelle des modules, leur ait entièrement échappé et que cette découverte ait été réservée à Landen qui d'ailleurs n'en a tiré qu'un médiocre parti et qui n'a pas même vu qu'elle fournissait une méthode très simple pour calculer par approximation les arcs de section conique.

On s'étonnera moins que la même découverte ait échappé à Euler, si on observe que la belle intégrale due à ce grand Géomètre, l'a conduit à comparer entre elles les diverses valeurs d'une même transcendante, comme on compare les arcs d'une même courbe, ce qu'il a fait avec une élégance et une généralité qui ne laissent rien à désirer. Mais on ne voit dans aucun de ses Mémoires, qu'il ait fait varier les constantes ou les paramètres de ses fonctions, et qu'il ait ainsi passé d'une courbe à une autre, comme on le fait dans les comparaisons qui dépendent de l'échelle des modules. ${ }^{106}$

Toute lecture est située et celle que Legendre fait du théorème de Landen est tributaire du projet général de constitution d'un calcul des arcs d'ellipse dont elle est l'une des pierres angulaires. Si, comme l'écrit Catherine Goldstein, "la postérité d'un théorème peut être non seulement multiple, mais même instaurer, du 'précurseur' alors constitué à ses descendants, des lignées contradictoires" ${ }^{107}$, à l'inverse le processus même de constitution d'un 'précurseur' dépend d'une configuration spécifique par laquelle un ensemble de conditions, concurremment mises en œuvre, permettent l'identification d'un contenu dans ses premières occurrences supposées. Dans le cas qui nous occupe, il est tout aussi légitime de regarder le théorème de Landen comme un aboutissement que comme un commencement, selon qu'on l'envisage dans le contexte des recherches de Maclaurin et de d'Alembert qu'il complète et perfectionne, ou dans le contexte correspondant à la lecture de Legendre. Vouloir absolument trancher, de manière décontextualisée, la question de savoir qui de Landen, Lagrange ou Legendre est "le premier" à dégager la transformation de Landen est vain et illusoire. En revanche, nous pouvons à bon droit chercher à montrer que la constitution de Landen comme 'précurseur', et le fait que Legendre se démarque aussi bien de Lagrange, dont les méthodes d'approximation sont présentées comme foncièrement différentes de celles qui conduisent aux échelles de modules, que d'Euler, qui ne se serait pas soucié de faire varier les paramètres des fonctions, ne sont pas des données disparates mais participent d'une seule et même configuration que nous avons tenté de mettre en lumière.

Entre 1811 et 1825, Legendre remanie quelque peu le texte de son introduction historique, en supprimant des périodes entières et en en ajoutant d'autres. Si, d'un traité à l'autre, les inflexions ne sont le plus souvent que très légères, les suppressions qui concernent Lagrange et Landen ${ }^{108}$ sont toutefois significatives parce qu'elles effacent du texte définitif les traces du processus d'élaboration commencé dans les mémoires Sur les intégrations par arcs d'ellipse, contribuant ainsi à la fois à donner l'impression d'une sorte de préformation des contenus mathématiques chez les 'précurseurs' supposés, et à susciter des interrogations quant aux raisons qui expliquent que d'autres aient échoué à les discerner. Legendre est en effet beaucoup plus explicite dans la

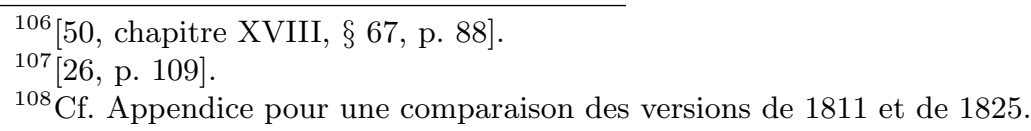


version de 1811. Il reconnaît ainsi sans ambages que la méthode de Lagrange permet de "ramener, par des transformations successives, l'intégrale $\int \frac{P d x}{R}$ à l'intégrale d'une formule semblable qui, par la disposition de ses coefficients, est facile à évaluer par approximation", et il ajoute que "ces transformations ont le double but de servir à la comparaison d'une suite de transcendantes formées d'après la même loi, et de conduire aux approximations les plus rapides dont ces fonctions sont susceptibles" ${ }^{109}$. Mais dans le même temps, il signale aussi n'être parvenu de son côté aux échelles de modules que parce qu'il s'était attaché à retrouver le théorème de Landen à partir de la méthode originale fondée sur la variation continue des paramètres qu'il avait mise en ouvre avant même d'avoir pris connaissance de ce théorème. Que, malgré leur parenté, les démarches respectives de Lagrange et de Legendre doivent néanmoins être distinguées aux yeux de ce dernier, résulte par conséquent de la même configuration spécifique qui l'amène aussi à proposer une démonstration nouvelle du résultat de Landen. En appliquant en effet, comme nous l'avons vu, les transformations successives que sa méthode générale stipule à la différentielle correspondant à l'élément d'arc d'ellipse, Lagrange produit sans doute lui aussi une suite échelonnée de transcendantes formées l'une à partir de l'autre "d'après une même loi", mais la suite ainsi obtenue déroge cependant aux exigences propres à un calcul des arcs d'ellipse, telles que Legendre les conçoit, pour autant que cette méthode conduit à des différentielles irrationnelles qui ne correspondent plus à des ellipses. Parallèlement, Legendre définit l'esprit de sa méthode en l'opposant à une approche, qu'il présente comme celle d'Euler, en choisissant de ne mettre en relief dans les recherches de ce dernier que celles qui confortent son interprétation, et qui, selon lui, exclurait la variation des paramètres parce qu'elle serait essentiellement préoccupée de la comparaison des arcs d'une même courbe.

Dans sa correspondance avec Jacobi, Legendre exprime très clairement la motivation profonde l'ayant animé tout au long de ses recherches sur les transcendantes elliptiques, à l'occasion d'une remarque que Jacobi lui fait au sujet de la dissymétrie entre les deux classes de fonctions de troisième espèce, logarithmiques et circulaires, laquelle est masquée tant qu'on fait abstraction de l'évaluation numérique et qu'on ne distingue pas entre les valeurs réelles et imaginaires, mais surgit dès lors que l'on se soucie de chercher une valeur approchée des transcendantes.

Je suis fâché de perdre l'espérance de réduire en table les fonctions à paramètre circulaire et j'ai peine à comprendre comment il peut y avoir une différence aussi essentielle entre les deux classes. Mais comme vous dites, cela tient à la nature des choses et nous ne pouvons rien y changer. Vous vous en consolez plus aisément que moi, vous et M. Abel qui êtes tous deux éminemment spéculatifs, mais moi qui ai toujours eu pour but d'introduire dans le calcul de nouveaux éléments qu'on puisse réaliser en nombres à volonté, moi qui me suis livré à un travail des plus longs et des plus fastidieux pour la construction des tables, travail que je n'hésite pas à croire aussi considérable que celui des grandes tables de Briggs, je ne prends pas mon parti aussi facilement sur l'espérance déçue que vous m'aviez fait concevoir, et dont une moitié seulement s'est réalisée. ${ }^{110}$

Pour autant que la méthode de variation continue des mémoires Sur les intégrations par arcs d'ellipse de 1786 témoigne de manière exemplaire de ces exigences conjointes du calcul numérique et de la nomenclature ordonnée qui caractérisent en propre le style des mathématiques de Legendre, le processus d'identification de la transformation analytique implicite dans le théorème de Landen n'est rien d'autre que le processus même d'une lecture orientée.

${ }^{109}[49$, p. 3].

${ }^{110}$ Cf. [51, p. 445], lettre à Jacobi du 4 juin 1829. 


\section{Appendice}

\section{Les présentations historiques de Legendre dans les Exercices de calcul intégral (1811) et le Traité des fonctions elliptiques (1825)}

Nous reproduisons ci-après le texte des présentations historiques que Legendre donne dans les Exercices et dans le Traité, en indiquant aussi bien les passages qui figurent dans la version de 1825, mais sont absents de celle de 1811 (signalés en caractères gras entre crochets droits), que ceux qui ont été supprimés entre la première et la seconde version (signalés en italiques entre parenthèses).

(Après avoir épuisé les formules différentielles qui s'intègrent tant algébriquement que par arcs de cercle ou par logarithmes, les Géomètres s'occupèrent de rechercher toutes celles qui sont intégrables par les arcs d'ellipse ou par les arcs d'hyperbole*...Les formules qu'on peut intégrer par cette voie se trouvèrent très-nombreuses; mais il n'y avait point de liaison entre les résultats, et ils étaient loin de former une théorie.) [Maclaurin et d'Alembert* sont les premiers qui se soient occupés des intégrales qui peuvent être exprimées par des arcs d'ellipse ou par des arcs d'hyperbole; ils trouvèrent un grand nombre de formules susceptibles de cette réduction, mais les différents résultats n'étaient point liés entre eux et ne pouvaient former aucune théorie.]

Un Géomètre italien d'une grande sagacité, ouvrit la route à des spéculations plus profondes ${ }^{\dagger}$. Il prouva que sur toute ellipse ou sur toute hyperbole donnée, on peut assigner, d'une infinité de manières, deux arcs dont la différence soit égale à une quantité algébrique. Il démontra en même temps que la [courbe nommée] lemniscate jouit de cette singulière propriété, que ses arcs peuvent être multipliés ou divisés algébriquement, comme les arcs de cercle, quoique chacun d'eux soit une transcendante d'un ordre supérieur. [; c'est le premier exemple où l'on ait montré l'usage de la plus simple des fonctions elliptiques, qui est en quelque sorte la régulatrice de toutes les autres et qui peut se transformer d'une infinité de manières sans cesser d'être semblable à elle-même.]

Euler, par une combinaison qu'on peut regarder comme fort heureuse, quoique ces ( $h a-$ sards) [hazards] n'arrivent (jamais) qu'à ceux qui savent les faire naître, trouva l'intégrale algébrique complète d'une équation différentielle composée de deux termes séparés, mais semblables, dont chacun n'est intégrable que par des arcs de sections coniques ${ }^{\ddagger}$.

Cette découverte importante donna lieu à son auteur de comparer d'une manière plus générale qu'on ne l'avait fait avant lui, non seulement les arcs d'une même ellipse (ou) [,] d'une même hyperbole,[ ou d'une même lemniscate,] mais en général toutes les transcendantes contenues dans la formule $\int \frac{P d x}{R}$, où $P$ est une fonction rationnelle de $x$, et $R$ (un radical de la forme $\sqrt{\alpha+\beta x+\gamma x^{2}+\delta x^{3}+\epsilon x^{4}}, \alpha, \beta, \gamma, \delta, \epsilon$ étant constans.) [la racine quarrée d'un polynome en $x$ du quatrième degré.]

(L'intégrale trouvée par Euler était trop remarquable pour ne pas fixer particulièrement l'attention des Géomètres.) Lagrange voulut faire rentrer (cette intégration) dans les procédés ordinaires de l'analyse [l'intégrale trouvée par Euler ${ }^{\S}$ ]; il y (réussit) [parvint] par une méthode fort ingénieuse dont l'application s'élève graduellement des transcendantes inférieures aux transcendantes Eulériennes, mais il (essaia) [essaya] inutilement de parvenir à un résultat plus général que celui d'Euler.

Peu de temps après, Landen, géomètre anglais, démontra que tout arc d'hyperbole peut être mesuré par deux arcs d'ellipse ; découverte mémorable qui (réduit aux seuls arcs d'ellipse toutes les intégrales qu'on n'avait pu exprimer jusques-là que par la rectification des

\footnotetext{
*Maclaurin, Traité des fluxions. - D'Alembert. Mém. de Berlin, 1746.

${ }^{\dagger}$ Fagnani. Produzioni matematiche, tom. II, 1750.

${ }^{\ddagger}$ Euler. Novi Com. Petrop., tom. VI et VII, 1761.

${ }^{\S}$ Mém. de Turin, tom. IV, 1768.

๑Philosophical Transactions, 1775, Mathematical Memoirs, by John Landen, 1780.
} 
deux courbes.) [simplifie la théorie des transcendantes, et qui aurait pu conduire l'auteur à d'autres résultats plus importans.]

Enfin Lagrange se signala à nouveau dans la même carrièrell en donnant une méthode générale pour (ramener, par des transformations successives, l'intégrale $\int \frac{P d x}{R}$ à l'intégrale d'une formule semblable qui, par la disposition de ses coefficients, est facile à évaluer par approximation.) [trouver par approximation les intégrales de la forme $\int \frac{P d x}{R}$.] (Ces transformations ont le double but de servir à la comparaison d'une suite de transcendantes formées d'après la même loi, et de conduire aux approximations les plus rapides dont ces fonctions sont susceptibles.)

Telles étaient les principales découvertes des Géomètres dans la théorie (des transcendantes désignées par $\int \frac{P d x}{R}$ ) [de ces intégrales], lorsque je publiai mes recherches sur l'intégration par arcs d'ellipse** [, où après avoir démontré la plupart des théorèmes connus jusqu'à cette époque, je fis voir que dans une suite infinie d'ellipses formées d'après une même loi, on peut réduire la rectification de l'une de ces ellipses à celle de deux autres prises à volonté dans la même suite.] (La première partie avait été composée avant que j'eusse connaissance du théorème de Landen; elle contenait des vues nouvelles sur l'usage des arcs d'ellipse, et particulièrement un moyen d'éviter l'emploi des arcs d'hyperbole dans le calcul intégral, en y suppléant par une table d'arcs d'ellipse dressée convenablement. Je donnai ensuite une nouvelle démonstration du théorème de Landen, et je prouvai par la même méthode, que toute ellipse donnée fait partie d'une suite infinie d'ellipses tellement liées entre elles, que par la rectification de deux de ces ellipses, prises à volonté, on obtient la rectification de toutes les autres. Ces ellipses ayant un demigrand axe commun égal à l'unité, et leurs excentricités variant suivant une loi connue, depuis zéro jusqu'à l'unité, on peut par ce théorème réduire la rectification d'une ellipse donnée à celle de deux autres ellipses aussi peu différentes du cercle qu'on voudra.) C'était un pas de plus dans une carrière difficile.

[49, Introduction, p. 1-3] [50, Introduction, p. 1-3]

\section{Références}

[1] G. Almkvist et B. Berndt - « Gauss, Landen, Ramanujan, the Arithmetic-Geometric Mean, Ellipses, $\pi$ and the Ladies Diary », The American Mathematical Monthly 95 (1988), no. 7, p. 585-608.

[2] É. D. Beaumont - Éloge historique de Adrien-Marie Legendre, Firmin-Didot, Paris, 1861.

[3] P. Bourdieu - Quelques propriétés des champs, 1976/2002, Exposé à l'ENS, novembre 1976. In Questions de sociologie, pp. 113-120. Paris : Les Editions de Minuit, 2002.

[4] F. CAJORI - A history of mathematics, Macmillan, New-York, 1894, Seconde édition élargie, 1919.

[5] - , A history of the conceptions of limits and fluxions in Great Britain from Newton to Woodhouse, Open Court, Chicago-Londres, 1919.

[6] M. Cantor - Vorlesungen über Geschichte der Mathematik, vol. IV, Teubner, Leipzig, 1908.

[7] F. Casorati - Teorica delle funzioni di variabili complesse, vol. I, Fusi, Pavia, 1868.

[8] A. Cayley - «Landen », (1882), in Encyclopedia Britannica, 9ème édition, 14, p. 271. Repris dans les Collected Works, vol. XI, art. 791, pp. 583-4.

[9] R. Cooke - «Elliptic integrals and functions », dans Companion Encyclopedia of the History and Philosophy of the Mathematical Sciences, Grattan-Guinness, I. (éd.), vol. 1, Routledge, 1994, pp. 529-539.

\footnotetext{
"Nouveaux Mémoires de Turin, an. 1784 et 1785, tom. II.

** Mém. de l'Acad. des Sciences de Paris, ann. 1786.
} 
[10] D. A. Cox - « The arithmetic-geometric mean of Gauss », L'enseignement mathématique 30 (1984), p. 275-330.

[11] J. L. R. D'AlemberT - « Recherches sur le calcul intégral », Histoire de l'Académie royale des sciences et des belles-lettres de Berlin (année 1748) (1746), p. 182-224, Réédition dans [12, p. 99-164].

[12] —, Euvres complètes. Série I. Traités et mémoires mathématiques, 1736-1756. vol. 4a. textes de mathématiques pures (1745-1752), CNRS éditions, Paris, 2007, Édition établie par C. Gilain.

[13] J.-B. Delambre - « Notice sur la vie et les ouvrages de M. le Comte J.-L. Lagrange », (1867), dans [40, tome I, pp. IX-LI].

[14] A. EnnePer - Elliptische Functionen. Theorie und Geschichte, Halle, 1876, 2e éd. par F. Müller, Halle, 1890.

[15] L. EULER - « De integratione æquationis differentialis $\frac{m d x}{\sqrt{1-x^{4}}}=\frac{n d y}{\sqrt{1-y^{4}}} »$, Novi comm. acad. sci. Petrop. 6 ((1756/7) 1761), p. 37-57, repris dans [19, E 251. p. 58-79].

[16] — , «Observationes de comparatione arcuum curvarum irrectificabilium », Novi comm. acad. sci. Petrop. 6 ((1756/7) 1761), p. 58-84, repris dans [19, E 252. p. 80-107].

[17] — , «Specimen novæ methodi curvarum quadraturas et rectificationes aliasque quantitates transcendentes inter se comparandi », Novi comm. acad. sci. Petrop. 7 ((1758/9) 1761), p. 83-127, repris dans [19, E 263. p. 108-152].

[18] —, « De reductione formularum integralium ad rectificationem ellipsis ac hyperbolæ », Novi comm. acad. sci. Petrop. 10 ((1764) 1766), p. 3-50, repris dans [19, E 295. p. 256-301].

[19] — , Opera omnia. Ser. I, vol. 20. Commentationes analytica ad theoriam integralium ellipticorum pertinentes, vol. 1, Teubner, Leipzig, 1912, édition établie par A. Krazer.

[20] G. C. FAgnANO - « Teorema da cui si deduce una nuova misura degli archi elittici, iperbolici e cicloidali », Giornale de' Letterati d'Italia 26 (1716), p. 266 et suivantes, repris dans [21, II, pp. 336-342].

[21] — , Produzioni matematiche, Pesaro, 1750, 2 vols, réédition dans [22, vol. I \& II].

[22] —, Opere matematiche del marchese Giulio Carlo de' Toschi e Sant'Onofreo di Fagnano, Milan-Rome-Naples, 1911-1912, Volterra, V., Loria, G. et Gambioli, D. (éds). 3 vol.

[23] R. FRICKE - «Elliptische Funktionen », (1913), dans Encyklopädie der mathematischen Wissenschaften mit Einschluss ihrer Anwendungen, Leipzig, Teubner, 1898-1935, article II B 3 .

[24] C. Gilain - « D'Alembert et l'intégration des expressions différentielles à une variable », (2002), dans Paty, M. \& Michel, A. (éds), Analyse et dynamique. Études sur l'œuvre de d'Alembert, Presses de l'Université de Laval, Canada, 207-235.

[25] — , «Introduction générale », (2007), dans Euvres complètes. Série I. Traités et mémoires mathématiques, 1736-1756. Vol. 4a. Textes de mathématiques pures (1745-1752), édition établie par C. Gilain, pp. xiii-cvii.

[26] C. Goldstein (éd.) - Un théorème de Fermat et ses lecteurs, Presses Universitaires de Vincennes, Saint-Denis, 1995.

[27] C. Goldstein et N. Schappacher - « A book in search of a discipline (1801-1860)», (2007a), dans The Shaping of Arithmetic after C. F. Gauss's Disquisitiones Arithmeticæ, Berlin-Heidelberg, Springer, 3-65.

[28] J. Gray - «A commentary on Gauss's mathematical diary, 1796-1814, with an English translation », Expos. Math. 2 (1984), p. 97-130. 
[29] N. Guicciardini - The development of Newtonian calculus in Britain. 1700-1800, Cambridge University Press, Cambridge, 1989.

[30] H. Gwynedd Green et H. J. J. Winter - « John Landen, F. R. S. (1719-1790)», Isis 35 (1944), no. 1, p. 6-10.

[31] C. Houzel - « Fonctions elliptiques et intégrales abéliennes », (1978), Abrégé d'Histoire des Mathématiques 1700-1900, réédition 1992, Paris, Hermann, p. 293-314.

[32] — , La géométrie algébrique. Recherches historiques, Blanchard, Paris, 2002.

[33] C. G. J. JACOBI - « Extrait d'une lettre adressée à M. Hermite », Journal für die reine und angewandte Mathematik 32 (1845), p. 176-181.

[34] — , Gesammelte Werke, Reimer, Berlin, 1881-1891, éd. C. W. Borchardt.

[35] M. KLINE - Mathematical thought from ancient to modern times, Oxford University Press, New-York, 1972.

[36] L. Koenigsberger - Zur Geschichte der Theorie der elliptischen Transcendenten in den Jahren 1826-29, Teubner, Leipzig, 1879.

[37] A. Krazer - «Zur Geschichte des Umkehrproblems der Integrale », Jahresbericht der deutschen Mathematiker-Vereinigung 18 (1909), p. 44-75.

[38] — , «Vorwort des Herausgebers », (1912), dans [19, p. vii-x].

[39] J.-L. LAGRANGE - « Sur une nouvelle méthode de calcul intégral, pour les différentielles affectées d'un radical carré sous lequel la variable ne passe pas le quatrième degré », Mémoires de l'Académie royale des sciences de Turin II (1784-1785/1786), Euvres, II, pp. 253-312.

[40] — , Euvres de Lagrange, vol. I-XIV, Gauthier-Villars, Paris, 1867-1892, publiées par les soins de J.-A. Serret et G. Darboux.

[41] J. LANDEN - Mathematical lucubrations, 1755.

[42] — , A discourse concerning residual analysis : a new branch of the algebraic art, of very extensive use, both in pure mathematics and natural philosophy, Nourse, London, 1758.

[43] —, The residual analysis, 1764.

[44] J. LANDEN - «A disquisition concerning certain fluents, which are assignable by the arcs of the conic sections; wherein are investigated some new and useful theorems for computing such fluents », Philosophical Transactions of the Royal Society of London 61 (1771), p. 298309 .

[45] — , « An investigation of a general theorem for finding the length of any arc of any conic hyperbola, by means of two elliptic arcs, with some new and useful theorems deduced therefrom », Philosophical Transactions of the Royal Society of London 65 (1775), p. 283289.

[46] A.-M. Legendre - " Mémoire sur les intégrations par arcs d'ellipse », Histoire de l'Académie Royale des Sciences (1786a), p. 616-643.

[47] — , « Second mémoire sur les intégrations par arcs d'ellipse, et sur la comparaison de ces arcs », Histoire de l'Académie Royale des Sciences (1786b), p. 644-683.

[48] — , Mémoire sur les transcendantes elliptiques, Du Pont \& Firmin Didot, Paris, 1793.

[49] A. M. LEGENDRE - Exercices de calcul intégral sur divers ordres de transcendantes et sur les quadratures, vol. 1, Courcier, Paris, 1811.

[50] — , Traité des fonctions elliptiques et des intégrales eulériennes avec des tables pour en faciliter le calcul numérique, vol. I, Huzard-Courcier, Paris, 1825. 
[51] A. M. Legendre et C. G. J. JaCobi - « Correspondance mathématique », Journal für die reine und angewandte Mathematik 80 (1875), p. 205-279, Reproduite dans [34, I, p. 390-461].

[52] C. Maclaurin - « Tractatus de curvarum constructione \& mensura; ubi plurimæ series curvarum infinitæ vel rectis mensurantur vel ad simpliciores curvas reducuntur », Philosophical Transactions of the Royal Society of London) 30 (1720), p. 803-811.

[53] — , A treatise of fluxions, vol. 2 vol., Edinburgh, 1742, Trad. française par R. P. Pézenas, Paris, Jombert, 1749.

[54] P. MAnsion - Théorie de la multiplication et de la transformation des fonctions elliptiques. Essai d'exposition élémentaire, Gauthier-Villars, Paris, 1870.

[55] M. MARIE - Histoire des sciences mathématiques et physiques. Vol. viii. D'Euler à Lagrange, Gauthier-Villars, Paris, 1886.

[56] J.-E. Montucla - Histoire des mathématiques, 2ème éd., vol. 3, Lalande, Paris, 1802.

[57] F. J. Richelot - Die Landen'sche Transformation in ihrer Anwendung auf die Entwickelung der elliptischen Funktionen, Hübner et Matz, Königsberg, 1868.

[58] C. L. Siegel - «Zur Vorgeschichte des Eulerschen Additionstheorems », (1956/1959), dans Sammelband Leonhard Euler, Akademie-Verlag Berlin. Réédition dans Gesammelte Abhandungen), vol. III. 249-251.

[59] I. Smadja - « De la lemniscate au damier analytique. Legendre et le primat de l'analyse », Oriens-Occidens $\mathbf{8}$ (2010).

[60] R. Taton - «Inventaire chronologique de l'œuvre de Lagrange », Revue d'histoire des sciences 41 (1974), no. 1, p. 39-74.

[61] — , « Le départ de Lagrange de Berlin et son installation à Paris en 1787 », Revue d'histoire des sciences 27 (1988), no. 1, p. 3-36.

[62] G. Vivanti - «Infinitesimalrechnung », (1908), dans [6], section XXVI, pp. 639-869, et en particulier "Transcendenten. Elliptische Integrale", pp. 790-866.

[63] G. N. Watson - " The marquis and the land-agent : a tale of the eighteenth century », The Mathematical Gazette 17 (1933), no. 222, p. 5-17.

[64] A. WeIL - Number Theory. An Approach through History from Hammurapi to Legendre, Birkhaüser, Boston, 1984. 